Portland State University

PDXScholar

$12-2-1994$

\title{
Predicting Solute Transport in Natural Streams - A Stochastic Approach
}

Xueqing Zhou

Portland State University

Follow this and additional works at: https://pdxscholar.library.pdx.edu/open_access_etds

Part of the Civil Engineering Commons

Let us know how access to this document benefits you.

Recommended Citation

Zhou, Xueqing, "Predicting Solute Transport in Natural Streams - A Stochastic Approach" (1994).

Dissertations and Theses. Paper 5057.

https://doi.org/10.15760/etd.6933

This Thesis is brought to you for free and open access. It has been accepted for inclusion in Dissertations and Theses by an authorized administrator of PDXScholar. Please contact us if we can make this document more accessible: pdxscholar@pdx.edu. 


\section{THESIS APPROVAL}

The abstract and thesis of Xueqing Zhou for the Master of Science in Civil Engineering was presented December 2, 1994 and accepted by the thesis committee and the department.

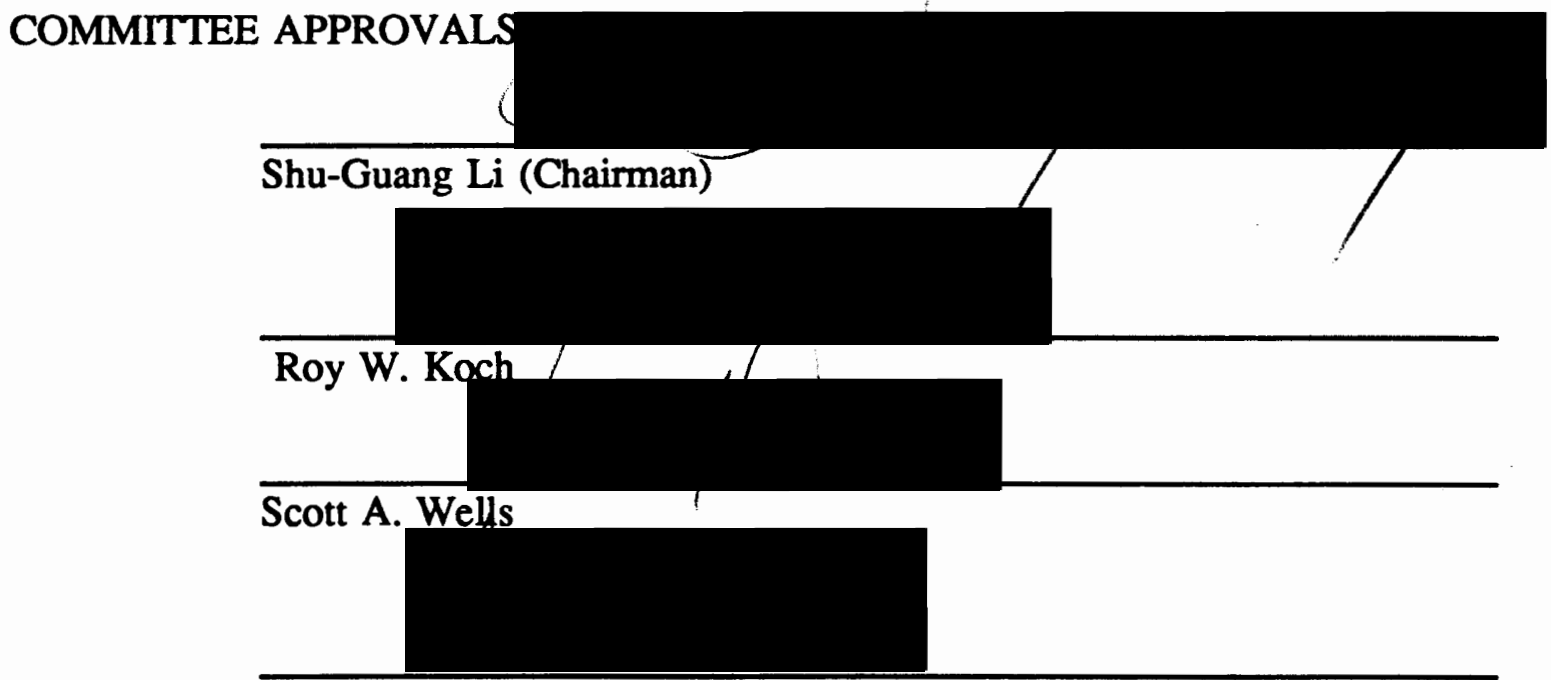

Scott F. Burns

(Representative of the Office of Graduate Studies)

DEPARTMENT APPROVALS:

Franz N. Rad, Chair

Department of Civil Engineering



ACCEPTED FOR PORTLAND STATE UNIVERSITY LIBRARY

by






\begin{abstract}
An abstract of the thesis of Xueqing Zhou for the Master of Science in Civil Engineering presented on December 2, 1994.
\end{abstract}

\title{
Title: Predicting Solute Transport in Natural Streams - A Stochastic Approach
}

The existing theories for predicting longitudinal dispersion in straight open channels have long been recognized as inadequate when applied to natural rivers. These theories tend to grossly underestimate dispersion in real streams since an important mixing mechanism due to nonuniform river cross-section variations is not explicitly taken into account.

Recognizing the important role of stream irregularities on solute transport and the analytical difficulties of classical deterministic analysis, we develop a stochastic approach for analyzing solute transport in natural streams. Variations in river width and bed elevation are conveniently represented as one-dimensional random fields, characterized by their autocorrelation functions. Advection and dispersion due to the combined effect of turbulent diffusion and nonuniform flow are described by the stochastic solute transport equation. When boundary variations are small and statistically homogeneous, a stochastic spectral technique is used to obtain closed-form stochastic solutions. In particular, closed-form expressions are obtained for effective mean solute transport velocity and effective dispersion coefficient reflecting mixing due to flow variations both within the river cross-section and in the streamwise direction. The results show that the mean behavior of solute transport in a statistically irregular stream can be described as a gradient dispersion process. The effective mean transport velocity in 
natural rivers is smaller than that in a corresponding uniform channel, and the effective longitudinal dispersion coefficient in natural rivers can be considerably greater than that of uniform open channels. The discrepancy between uniform channels and natural rivers increases rapidly as the variances of river width and bed elevation increase, especially when the mean flow Froude number is high. 


\title{
PREDICTING SOLUTE TRANSPORT IN NATURAL STREAMS
}

-- A STOCHASTIC APPROACH

\author{
by
}

XUEQING ZHOU

A thesis submitted in partial fulfillment of the requirements for the degree of

MASTER OF SCIENCE

in

CIVIL ENGINEERING

Portland State University

1995 


\section{Acknowledgments}

I wish to thank the members of my thesis committee, Professors Shu-Guang $\mathrm{Li}$ (Chairman), Scott Wells, Roy Koch and Scott Burns for their guidance. This thesis has been studied for about one year. During this one year, I have bothered my advisor, $\mathrm{Dr}$. Li, with all kinds of troubles and questions, his intelligent advising and encouragement have been invaluable to the study. He carefully and painstakingly read, with insightful comments on, every draft of this thesis. I also wish to express my appreciation to Professor Wells for his encouragement in my early graduate study.

The financial support from the Department of Civil Engineering at Portland State University is acknowledged, which offered me an opportunity to work on a fascinating project.

My many thanks to my classmates, especially Julie Peyton. She carefully read and edited three manuscripts, her comments have been very helpful.

Much gratitude is expressed to all of my friends. Dee Anne and Michael Bess carefully edited the first two chapters. Martha Samco checked the grammar for me chapter by chapter. I am grateful to the encouragements from Eleanor and Ted Watkins, Linda and Tom Davis.

I would especially like to acknowledge my family, whose encouragements and unconditional love have allowed me to pursue my goals. Finally, I owe special thanks to Zhongren Peng, my husband, for his consistent assistance and unwavering support. His unfailing love and encouragement have helped make this thesis possible. 


\section{Contents}

1 Introduction 1

2 A Critical Review of Previous Research 6

2.1 Analytical Approaches $\ldots \ldots \ldots \ldots \ldots$

2.1.1 Taylor's Theory . . . . . . . . . . . . . 7

2.1.2 Elder's Theory $\ldots \ldots \ldots \ldots \ldots \ldots$

2.1.3 Fischer's Theory . . . . . . . . . . . 10

2.2 Semi-Empirical Approaches $\ldots \ldots \ldots \ldots \ldots \ldots$

3 A Stochastic Approach for Predicting Solute Transport 14

3.1 Stochastic Concept . . . . . . . . . . . 15

3.1.1 Random Field Representation of Irregular Streams _. . . 15

3.1.2 Spectral Representation of Stationary Stochastic Processes . 20

3.2 Stochastic Transport Analysis . . . . . . . . . . . 24

3.2.1 Stochastic Solute Transport Equation . . . . . . . . . 24

3.2.2 Closed-Form Spectral Solutions $\ldots \ldots \ldots \ldots$. . . . . 31 
3.2.3 Effective Mean Transport Equation . . . . . . . . . 35

4 Discussion and Evaluation of Results 40

4.1 Effective Dispersion Coefficient $\ldots \ldots \ldots \ldots \ldots \ldots$

4.2 Effective Mean Transport Velocity . . . . . . . . . . . 49

4.3 Parameter Estimation $\ldots \ldots \ldots \ldots \ldots \ldots$

5 Conclusions $\quad 58$

$\begin{array}{ll}\text { A Notation } & 63\end{array}$ 


\section{List of Figures}

1-1 Definition sketch of different mixing zones $\ldots \ldots \ldots \ldots \ldots$

3-1 Summary of the stochastic approach. (Source: Gelhar, 1993, pp.

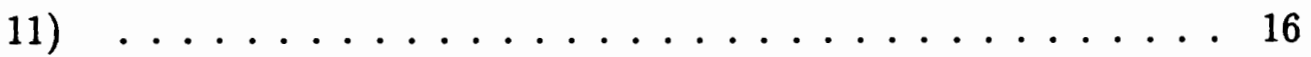

3-2 Definition sketch of a stream's geometric variation . . . . . . 17

3-3 A realization of a stochastic process and the ensemble with the embedded realization $\ldots \ldots \ldots \ldots \ldots \ldots \ldots$

3-4 Schematic representation of different types of covariance-spectrum

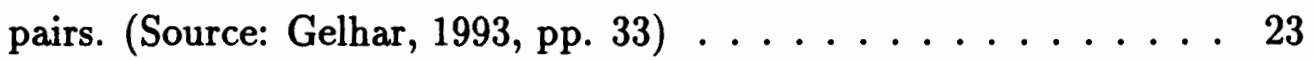

4-1 Contours of normalized effective longitudinal dispersion coefficient versus the coefficient of variation of river width and bed elevation . 43

4-2 Contours of coefficient $\alpha_{1}$ versus the Froude number and $\bar{U} \lambda_{B} / K_{0} .45$

4-3 Effect of $\Gamma_{B}$ on the coefficient $\alpha_{1}$ for different $F_{r} \ldots \ldots 47$

4-4 Effect of $\Gamma_{B}$ on the coefficient $\alpha_{1}$ for different $P_{B} \ldots \ldots 48$ 
4-5 Contours of normalized effective transport velocity versus the coefficient of variation of river width and bed elevation for $F_{r}=0.2 \ldots 50$

4-6 Contours of normalized effective transport velocity versus the coefficient of variation of river width and bed elevation for $F_{r}=0.4 \ldots 51$

4-7 Contours of normalized effective transport velocity versus the coefficient of variation of river width and bed elevation for $F_{r}=0.6 \ldots 52$

4-8 Effect of Froude number on the coefficients $\beta_{1}$ and $\beta_{2} \ldots \ldots$

4-9 Effect of $\bar{U} \lambda_{B} / K_{0}$ (denoted as $P_{B}$ ) on the coefficient $\beta_{1}$ (solid line); Effect of $\bar{U} \lambda_{z} / K_{0}$ (denoted as $P_{z}$ ) on the coefficient $\beta_{2}$ (dotted line) 55

4-10 Effect of $\bar{U} \lambda_{B} / K_{0}$ (denoted as $\left.\Gamma_{B}\right)$ on the coefficient $\beta_{1}$ (solid line); Effect of $\bar{U} \lambda_{z} / K_{0}$ (denoted as $\Gamma_{z}$ ) on the coefficient $\beta_{2}$ (dotted line) 56

5-1 Summary of the stochastic results of solute transport in natural

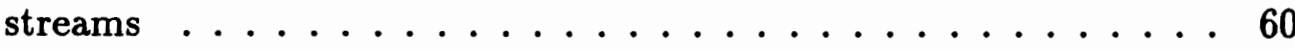




\section{List of Tables}

4.1 Parameter values used in Figures $4-1$ through $4-10 \ldots \ldots$. . . . 42 


\section{Chapter 1}

\section{Introduction}

It has been a common practice throughout the world to discharge waste materials into streams both intentionally and unintentionally. In order to better control the contamination of a stream and to predict levels of pollution, it is essential to have a good understanding of the rate at which a stream is capable of transporting and dispersing a pollutant. The receiving water quality in real streams depends on both the hydrodynamics of transport and mixing, as well as the chemistry and biology of natural water systems. This thesis deals with the hydrodynamics of transport, primarily mixing due to differential velocity distributions, in natural rivers.

The process of contaminant mixing in natural rivers can be divided into three stages, as illustrated in Figure 1-1. The first stage (A) involves the so-called "near field" mixing in the immediate neighborhood of the contaminant outlet. In this zone, solute transport is often fully three dimensional and strongly affected by 


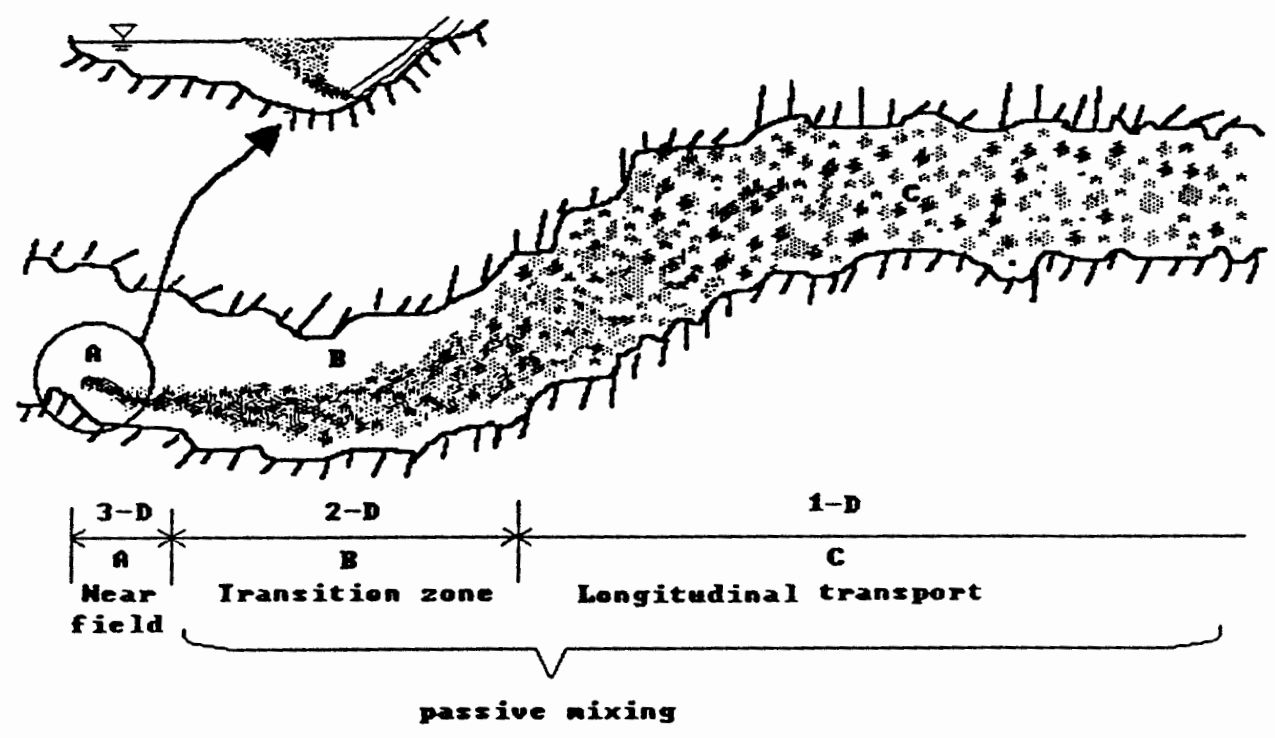

Figure 1-1: Definition sketch of different mixing zones

initial contaminant flow momentum and buoyancy (e.g., Crow et al. 1971; Fischer et al. 1979; Grant, 1974; Hirst, 1972; and Kizer, 1963).

As the solute is mixed and diluted, the effects of the initial momentum and buoyancy are dissipated. This leads to the subsequent second and third stages of "passive mixing" in which the combined effect of turbulence and advective currents (differential velocity) dictates. In the second stage (B), the solute is often fully mixed in the vertical direction and spreads mostly in the lateral direction.

The third stage (C), after the waste has adequately mixed across the channel, begins with the reduction of longitudinal concentration gradients by longitudinal dispersion. Unlike mixing in lakes and oceans, because of the relative narrowness of 
most streams, the solute transport is often predominantly in longitudinal direction. Comparing the first two stages with the last stage, mixing in the first two stages often occurs over a much shorter distance. The river zone where the last stage longitudinal transport - occurs is the focus area of this thesis.

Numerous investigations, beginning with Taylor $(1953,1954)$ and continuing with, for example, Aris (1956), Elder (1959), Fischer (1967, 1968, 1973), Sayre (1968), Sooky (1969), Atesman (1970), and Miller et al. (1974), have contributed to a good understanding of the mechanism of longitudinal dispersion in prismatic open channel flows. The cloud of dispersing material is spread over the channel cross-section by turbulent and advective mass transfer, and at the same time stretched longitudinally due to the variation of velocity with position in the crosssection. Given the magnitude and distribution of velocity and turbulent mass transfer coefficients in a longitudinally uniform flow, the existing theories of longitudinal dispersion are capable of predicting the dispersion coefficient and longitudinal concentration distribution with a high degree of accuracy over the entire span of the process.

In natural streams, however, comparable progress has not been achieved. The existing theories based on Taylor's $(1953,1954)$ analysis have long been recognized as being inadequate. The observed longitudinal dispersion coefficient seems almost always much larger than the theoretical predictions, in some cases by a factor of ten or more (Day, 1975; Nordin and Sabol, 1974; Godfrey and Frederick, 1970; Sooky, 
1969; Bansal, 1970; Chatwin, 1971). Even for some nominally straight natural rivers, the result of applying the previous theories is still found to be significantly different from the observed dispersion. Evaluation of this dispersion coefficient, in practice, therefore must rely in most cases on in-situ field observations (Nordin and Sabol, 1974; Godfrey and Frederick, 1970; Thackston and Krenkel, 1967), or calibration on a trial-and-error basis. These empirical or calibrated values, however, do not have any generality for geometric and flow conditions other than those for which data are collected or calibrations are performed, because geometries of natural rivers, varying from one to another, are not explicitly accounted for.

The discrepancies between the previous theories and field results are not surprising, as natural streams probably never meet the required assumption of uniform cross-section except for short stretches. The flow velocity is constantly and naturally changing on a large scale in the stream-wise direction in an irregular manner due to longitudinal "random" variations of river width and bed elevation. Predicting longitudinal dispersion in natural streams by using a traditional deterministic approach can, therefore, be far more difficult than predicting such in uniform open channels.

Recognizing that the additional important mechanism of mixing due to longitudinal "random" geometric variations precludes a conventional deterministic analysis, at least analytically, this thesis presents a stochastic approach for analyzing solute transport in irregular streams. In particular, the thesis aims at 
quantifying the effect of stream irregularity on solute transport.

This thesis is composed of five chapters. Chapter 2 reviews critically the existing methods for predicting longitudinal solute transport in streams and the motivation for the thesis research. Chapter 3 begins with a review of stochastic modeling concepts and stochastic processes and presents a stochastic theory for analyzing solute transport in irregular streams. Chapter 4 discusses and evaluates the stochastic analysis results. Finally, Chapter 5 summarizes the major findings and limitations of this research and presents recommendations for future research. 


\section{Chapter 2}

\section{A Critical Review of Previous}

\section{Research}

In this section, the state-of-the-art of knowledge in solute transport in natural streams is presented, and existing methods for predicting longitudinal solute transport are critically reviewed.

\subsection{Analytical Approaches}

Numerous researchers have contributed to the understanding of longitudinal solute transport in open channels and natural streams. The works by Taylor (1921, 1953, 1954), Elder (1959) and Fischer $(1967,1968,1979)$ represent three major milestones. The developmental process of these theories mirrors the development of research in longitudinal solute transport in natural streams. 


\subsubsection{Taylor's Theory}

The great English fluid mechanician, Sir Geoffrey Taylor, published his first classical paper in 1921, titled, "Diffusion by Continuous Movements." In this paper, Taylor analyzed spreading of a marked cloud in a homogeneous, stationary and one-dimensional turbulent fluid with zero mean velocity. Although the problem is not directly related to stream dispersion, the analysis laid down the theoretical framework for subsequent investigations of solute transport in streams. Taylor tackled this problem using a Lagrangian approach (Taylor, 1921) by relating the dispersion of a group of particles to the correlation between continuous displacements of individual random moving particles and obtained the following simple equation characterizing spreading of the cloud after large releasing time

$$
\sigma_{x}^{2}(t)=2 \sigma_{u}^{2} \lambda_{t} t
$$

where $\sigma_{u}^{2}$ is variance or mean-square-particle-velocity fluctuation, $t$ is time, $\lambda_{t}$ is the Lagrangian integral time scale of turbulence.

Equation (2.1) shows that, if one waits long enough, the variance of the particle cloud increases linearly with time at a rate given by $2 \sigma_{u}^{2} \lambda_{t}$. This formally justifies the attempt to model mixing due to turbulent fluctuations as a gradient diffusion process for reasonably large times after release, which eliminates the need to deal with the local details of turbulence. 
Taylor later extended the analysis to mixing of dissolved contaminants in both laminar (Taylor, 1953) and turbulent (Taylor, 1954) flow through a pipe. In the case that one is only interested in longitudinal transport, a solute particle can be considered to be moving at the mean velocity, with random displacements forward or backward caused by molecular and turbulent diffusion, and most importantly, by the differential velocities sampled by the particle as it moves up and down, back and forth randomly across the flow. Thus, one could view the particle's longitudinal velocity as a stationary, random function of time. By analogy with his 1921 analysis, Taylor was able to quantify that the longitudinal spread of a group of particles in pipe flow as a gradient dispersion process, once sufficient time has elapsed, and obtained the following formula for predicting the longitudinal dispersion coefficient,

$$
K_{0}=\frac{R^{2} \bar{U}^{2}}{48 D}, \text { for laminar flow }
$$

where $R$ is the pipe radius; $\bar{U}$ is the cross-sectional mean velocity; and $D$ is the molecular diffusion coefficient, and

$$
K_{0}=10.1 R u_{*}, \quad \text { for turbulent flow }
$$

where $u_{*}$ is the shear velocity, caused by the shear stress $\left(\tau_{0}\right)$, and calculated by $\sqrt{\tau_{0} / \rho}$. 
Although Taylor's theory does not directly apply to stream dispersion problems, it has provided a formal systematic framework for analyzing longitudinal solute dispersion induced by a differential velocity distribution. Taylor's theory has since been extended to address a variety of environmental flows and transport problems.

\subsubsection{Elder's Theory}

The most pertinent application of Taylor's theory and the longitudinal dispersion concept to free surface flows was performed by Elder (1959). Elder recognized that the only substantial difference between dispersion in a river and in a pipe lies in the different flow velocity profiles. Elder considered a wide river and assumed that longitudinal velocity varies only with the flow depth. Based on the well known logarithmic velocity profile (Fischer et al., 1979) and extending Taylor's theory, Elder obtained the following longitudinal dispersion coefficient for an open channel:

$$
K_{0}=5.93 \bar{u}_{*} \bar{h}
$$

where $\bar{h}$ is the mean flow depth, $\bar{u}_{*}$ is the mean shear velocity as defined previously. Elder's result represents the first step toward a better understanding of longitudinal dispersion in streams. 


\subsubsection{Fischer's Theory}

In trying to apply Elder's result for predicting solute transport in natural streams, however, Fischer (1966) found that Eqn. (2.4) grossly underestimates the actual longitudinal dispersion, sometimes by one or two orders of magnitude (Fischer, 1966).

Fischer attributed this discrepancy to Elder's assumption of uniform flow distribution across the flow. He realized that longitudinal dispersion in a real stream is in fact caused primarily by variation of velocity across the stream, rather than flow variation over depth. This is because, according to Taylor's theory, the longitudinal dispersion coefficient is proportional to the square of the distance over which the shear flow profile extends. A natural stream usually has a width to depth ratio in the range of ten or greater (Fischer et al., 1979). Thus the transverse profile should usually be considerably more important in producing longitudinal dispersion than the vertical profile.

Recognizing this and following Taylor, Fischer obtained the following equation, in terms of a general transverse velocity distribution across the flow, for predicting longitudinal dispersion in a natural stream (Fischer, 1969, Fischer et al., 1979),

$$
K_{0}=-\frac{1}{\bar{A}} \int_{\bar{y}_{l}}^{\bar{y}_{r}} U^{n}(\mu) h(\mu) \int_{\bar{y}_{l}}^{\mu} \frac{1}{E_{z} h(\eta)} \int_{\bar{y}_{l}}^{\eta} U^{n}(\xi) h(\xi) d \xi d \eta d \mu
$$

where $\bar{y}_{l}$ and $\bar{y}_{r}$ are the mean transverse coordinates of the left and right side of 
river bank; $h$ is the flow depth; $U^{n}$ is the velocity deviation from its cross-sectional mean; $\mu, \eta$, and $\xi$ are dummy integration variables; and $E_{z}$ is the turbulence and secondary flow induced transverse dispersion coefficient.

If the transverse velocity distribution is known in a stream, $K_{0}$ can be calculated by carrying out the triple integration in Eqn. (2.5). For a typical natural stream, $K_{0}$ predicted by Eqn. (2.5), is often much larger than that predicted by Elder's theory (Fischer et al., 1979).

\subsection{Semi-Empirical Approaches}

It must be emphasized at this point that the theories of Taylor, Elder and Fischer have all been based on the assumption of uniform prismatic channel cross-section. Turbulent diffusion and differential velocity distribution across the flow are the only mechanisms of longitudinal dispersion. A real stream is, however, characteristically irregular. Flow velocity also varies in the streamwise direction in a "random" fashion in response to irregular stream cross-sectional variations. This longitudinal flow nonuniformity leads to additional significant dispersion (Liu, 1977). As a result, Fischer's theory is also found to underestimate significantly the actual dispersion in streams (Fischer, 1967).

Recognizing the importance of stream irregularities and the analytical difficulties in using traditional deterministic approaches for predicting dispersion, practical engineers often resort to empirical or semi-empirical approaches for predicting 
solute transport. Based on a dimensional analysis of Eqn. (2.5) and available observed data, Fischer obtained the following semi-empirical equation for predicting longitudinal dispersion, without having to perform the detailed triple integrations in Eqn. (2.5):

$$
K=\frac{0.011 \bar{U}^{2} \bar{B}^{2}}{\bar{u}_{*} \bar{h}}
$$

where $\bar{U}$ is mean flow velocity, $\bar{B}$ is the mean channel width, $\bar{h}$ is the mean flow depth and $\bar{u}_{*}$ is the mean shear velocity. In most studies predictions by Eqn. (2.6) have been found to agree with observations within a factor of four or so (Fischer et al. 1979).

McQuivey and Keefer (1974), using a regressions analysis of field data, proposed the following simple formula for predicting longitudinal dispersion in streams:

$$
K=0.058 \frac{Q}{S B}
$$

where $Q$ is the discharge; $\bar{S}$ is the mean slope of the energy gradient; and $\bar{B}$ is the mean channel width.

Following Fischer, Liu (1977) further analyzed Eqn. (2.5), incorporated additional field data and obtained the following longitudinal dispersion coefficient:

$$
K=0.18\left(\frac{\bar{u}_{*}}{\bar{U}}\right)^{1.5} \frac{Q^{2}}{\bar{u}_{*} \bar{R}^{3}}
$$


where $\bar{R}$ is the cross-sectional mean hydraulic radius.

These semi-empirical equations have been used by many practitioners because of their simplicity. However, the coefficients and exponents in these equations are often "catch all" parameters calibrated from observed data. Such empirical values generally lack a physical basis and provide little physical insight into the dispersion processes.

In a nutshell, existing analytical approaches successfully predict longitudinal dispersion in prismatic channels caused by differential velocity distribution across the flow. However, these approaches do not take into account the additional mixing mechanism caused by cross-sectional variation and often grossly underestimate longitudinal dispersion in a natural stream. The empirically based approaches are site-specific, and can only be used for streams and flow conditions for which data are collected or calibrations are performed, because geometric variabilities of the natural river, varying from one river to another, are not explicitly accounted for.

It is highly desirable to have a systematic theoretical analysis of solute transport in natural streams that generalize the geometric irregularities and flow variation. 


\section{Chapter 3}

\section{A Stochastic Approach for}

\section{Predicting Solute Transport}

I have emphasized that natural rivers are characteristically irregular in crosssection and bed elevation. This kind of natural variability makes analyticaldeterministic analysis of solute transport extremely difficult, if not impossible. Here I adopt a probabilistic viewpoint and present a systematic, physically-based, stochastic analysis of solute transport in natural streams. 


\subsection{Stochastic Concept}

\subsubsection{Random Field Representation of Irregular Streams}

The stochastic approach applied in this thesis is based on the theory of random fields. The general idea is summarized schematically in Figure 3-1. The wellestablished continuum description of small-scale physics, which is captured by the classical partial differential equations (PDEs), is combined with a realistic but relatively simple probabilistic description of the stream geometric variability. Because the physical description is in a continuum form, the probabilistic description must be developed in a compatible fashion. That means the continuous variation of a random quantity in space needs to be described, and this requires the use of stochastic processes or random fields as will be discussed in the next paragraph. With random parameters or coefficients of this type, the classical continuum equations for transport become random or stochastic partial differential equations. These stochastic equations are then solved, usually in some approximate fashion, to produce the stochastic results.

The concept of a stochastic random process will be used to describe a stream's geometric variation (river width and bed elevation) in space. Figure 3-2 shows a schematic sketch of a "random" geometric variation in a stream. Where $B$ represents the river width, $z$ is the bed elevation averaged across the flow. I assume that the longitudinal variation of river width and bed elevation can be represented 




Figure 3-1: Summary of the stochastic approach. (Source: Gelhar, 1993, pp. 11) 

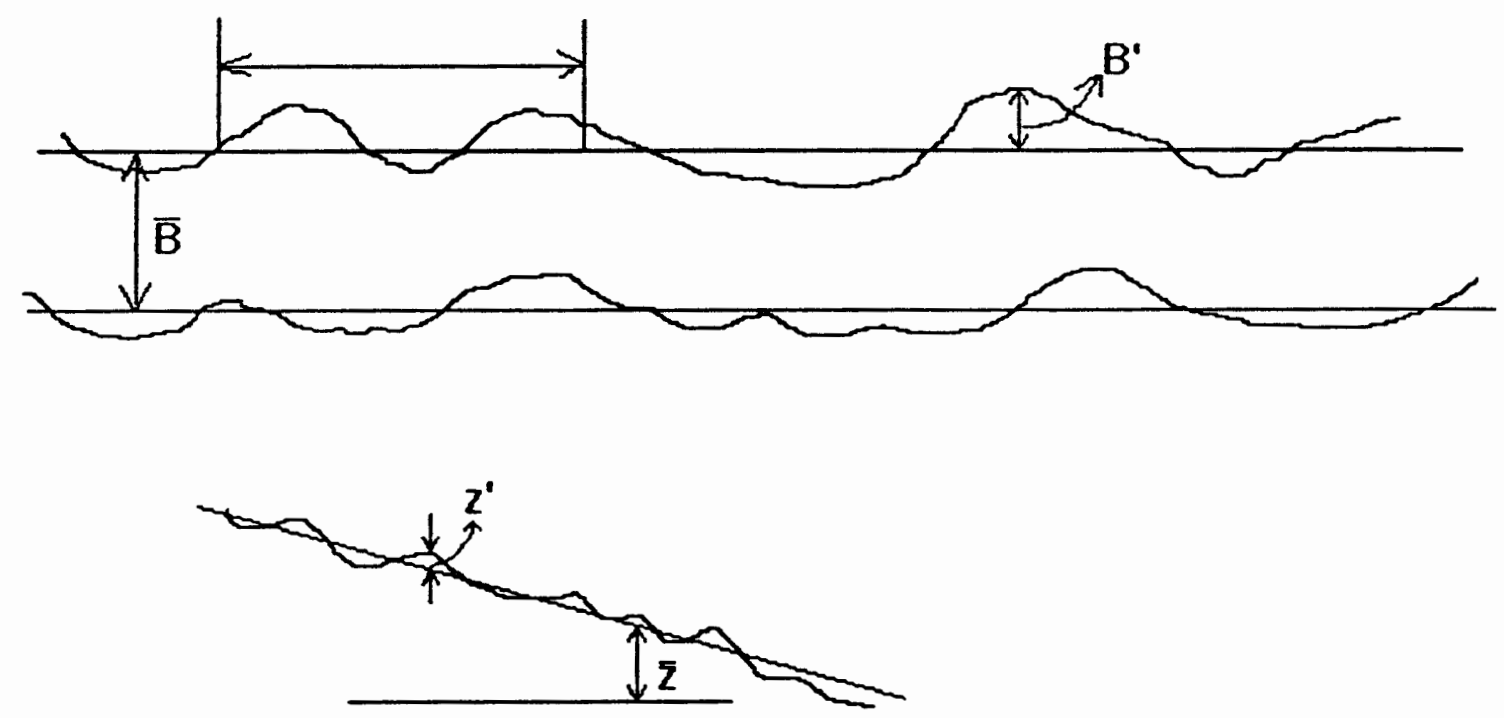

Figure 3-2: Definition sketch of a stream's geometric variation

as one-dimensional spatial random fields or stochastic processes, characterized by their variances $\left(\sigma_{B}^{2}, \sigma_{z}^{2}\right)$, mean $(\bar{B}, \bar{z})$ and correlation scale $\left(\lambda_{B}, \lambda_{z}\right)$. With the same statistic parameters, an infinite number of river realizations can be generated. The collection of all these possible realizations is called an stochastic ensemble. In most applications only one realization of the stream geometry will be available. Figure 3-3 illustrates the concept of the realization and the stochastic process. 

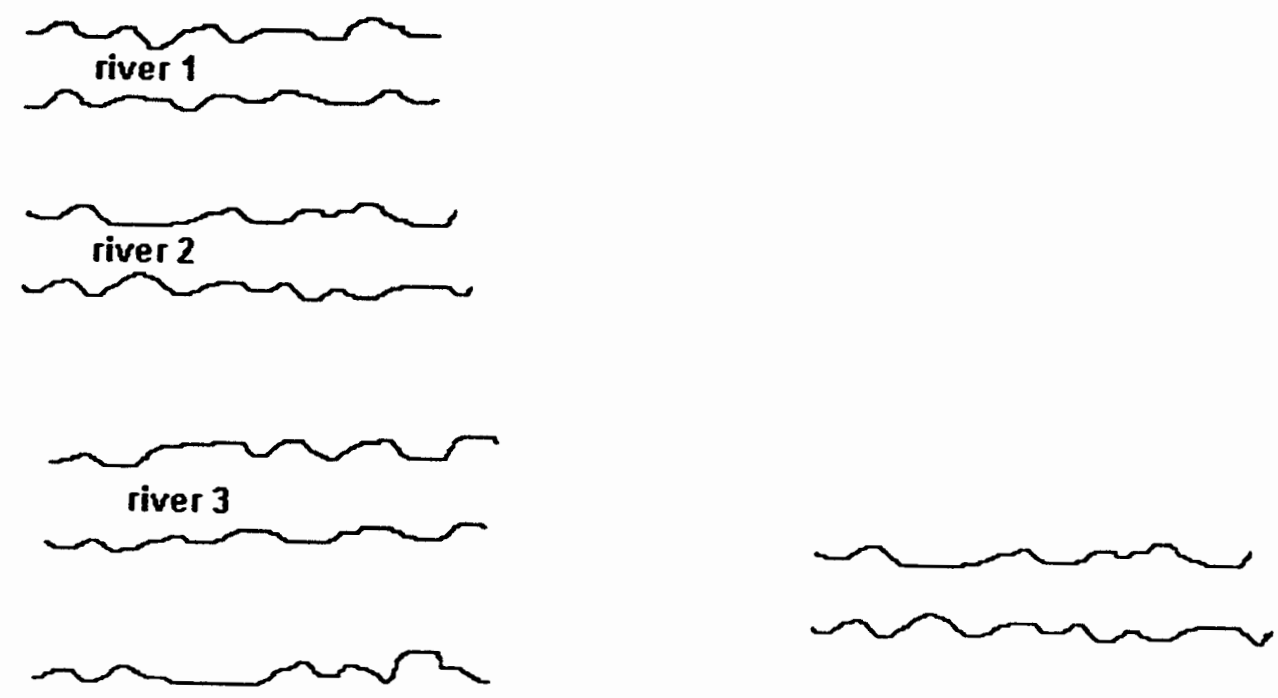

river 4



realization

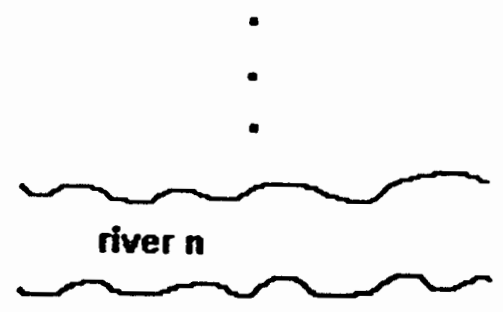

ensemble

Figure 3-3: A realization of a stochastic process and the ensemble with the embedded realization 
In order to analyze data from a single realization, it is often assumed that expected values or ensemble averages can be replaced by the realization's averages. This assumption is referred to as the ergodic hypothesis. In essence, I try to represent solute transport in a natural river with complex longitudinal variabilities in cross-section geometry (one realization of the stochastic process) by an ensemble average over a large number of realizations of the process, the latter being represented by the stochastic theory. Ergodicity requires, in this case, that the ensemble average be equivalent to a spatial average over some distance of the river. Lumey and Panofsky (1964) presented a pragmatic discussion of the ergodic question; they stated that the average distance must be several times the variability scale of the process for ergodicity to apply. For this reason the ensemble mean quantity, such as solution concentration derived in a stochastic analysis, is equivalent to the largescale spatial mean quantity averaged over a distance of several variability scales. Although ergodicity is difficult to prove, it is reasonable to expect that statistical analyses of single realizations will be approximations of ensemble statistics only if the overall length of the sample is larger than the correlation scale of the boundary variations. Note the means used in this thesis are all ensemble means. Based on the ergodic hypothesis the longitudinal means averaged cross-section represent ensemble means.

In order to apply the stochastic results to specific stream situations, the observation data must be incorporated. Through the process of statistical inference, nu- 
merical values for the parameters in a suitable probabilistic model, such as mean, variance and correlation functions of stream geometry, are evaluated systematically. When the results of statistical inference are introduced into the stochastic solutions, the behavior of a given stream situation is obtained.

\subsubsection{Spectral Representation of Stationary Stochastic Pro-}

\section{cesses}

A random function or stochastic process such as $f(x)$, representing the river width or bed elevation, can be considered statistically homogeneous or statistically uniform if the river width or bed elevation has a constant mean and its autocorrelation function depends only on the separation distance, $\zeta=x-x^{\prime}$. Physically, this homogeneity indicates that the degree of variability and the correlation structure do not depend on the spatial location. In this case, it is possible to represent the stationary mean-removed process $f^{\prime}(x)=f(x)-\bar{f}$ as a stochastic Fourier integral (sometimes referred to as a Stieltjes integral) (Priestley 1981)

$$
f^{\prime}(x)=\int_{-\infty}^{\infty} e^{i \omega x} d Z_{f}(\omega)
$$

where $\omega$ is the wave number, and $d Z_{f}(\omega)$ is the complex amplitude that satisfies the following orthogonality property 


$$
\overline{d Z_{f_{1}}(\omega) d Z_{f_{2}}^{c}\left(\omega^{\prime}\right)}=S_{f_{1} f_{2}}\left(\omega^{\prime}\right) \delta\left(\omega^{\prime}-\omega\right) d \omega d \omega^{\prime}
$$

where $\delta()$ is the Dirac delta function, $f_{1}$ and $f_{2}$ are arbitrary stationary random functions, the superscript $c$ denotes the complex conjugate, and $S_{f_{1} f_{2}}\left(\omega^{\prime}\right)$ is the cross-spectral density function or power spectrum. This spectral representation is unique and provides a convenient formalism for treating stationary random variables. The cross covariance function of two zero-mean real-valued stationary processes $f_{1}(x)$ and $f_{2}(x)$ can be written in terms of the inverse Fourier transform of the spectrum as follows

$$
R_{f_{1} f_{2}}(\zeta)=\overline{f_{1}^{\prime}(x) f_{2}^{\prime}(x+\zeta)}=\int_{-\infty}^{\infty} e^{i \omega \zeta} S_{f_{1} f_{2}}(\omega) d \omega
$$

when $f_{1}=f_{2}=f$, the covariance function of $f(x)$ can be written

$$
R_{f f}(\zeta)=\int_{-\infty}^{\infty} e^{i \omega \zeta} S_{f f}(\omega) d \omega
$$

and it reduces to the variance, $\sigma_{f}^{2}$, when $\zeta=0$.

$$
\sigma_{f}^{2}=\int_{-\infty}^{\infty} S_{f f}(\omega) d \omega
$$

Where $S_{f f}$ is the spectral density function of $f(x)$.

These results are the classical Fourier transform relationships between the covari- 
ance, variance and the spectrum. A commonly used covariance for describing natural variability is the following so-called "hole" exponential function (Gelher, 1993)

$$
R_{f f}(\zeta)=\sigma_{f}^{2}\left(1-\frac{|\zeta|}{\lambda}\right) e^{-\frac{|\zeta|}{\lambda}}
$$

which corresponds to the following power spectrum

$$
S_{f f}(\omega)=\frac{4 \sigma_{f}^{2}}{3 \pi^{2}} \frac{\omega^{3} \lambda^{3}}{\left(1+\omega^{2} \lambda^{2}\right)^{3}}
$$

where $\sigma_{f}^{2}$ and $\lambda$, respectively, are the variance and integral scale of the $f(x)$ process.

Several types of covariance-spectrum pairs are shown in Figure 3-4 along with typical realizations for each. The simple exponential covariance has the maximum spectral amplitude at zero frequency. The harmonic spectral amplitude does not exist as an ordinary function. The white noise covariance has the property that adjacent points in space are uncorrelated for arbitrarily small separation, the spectrum is constant and contains all frequencies. The "hole" type covariance-spectrum pair combines some elements of the simple exponential and the harmonic types. The correlation goes to zero for a large lag, but there is also implied some quasicyclic structure that leads to a small negative correlation at larger separation. This type of spectrum is different from the simple exponential type. The maximum spectral amplitude occurs at the middle frequency, while at lower and higher 


$$
f(x)
$$
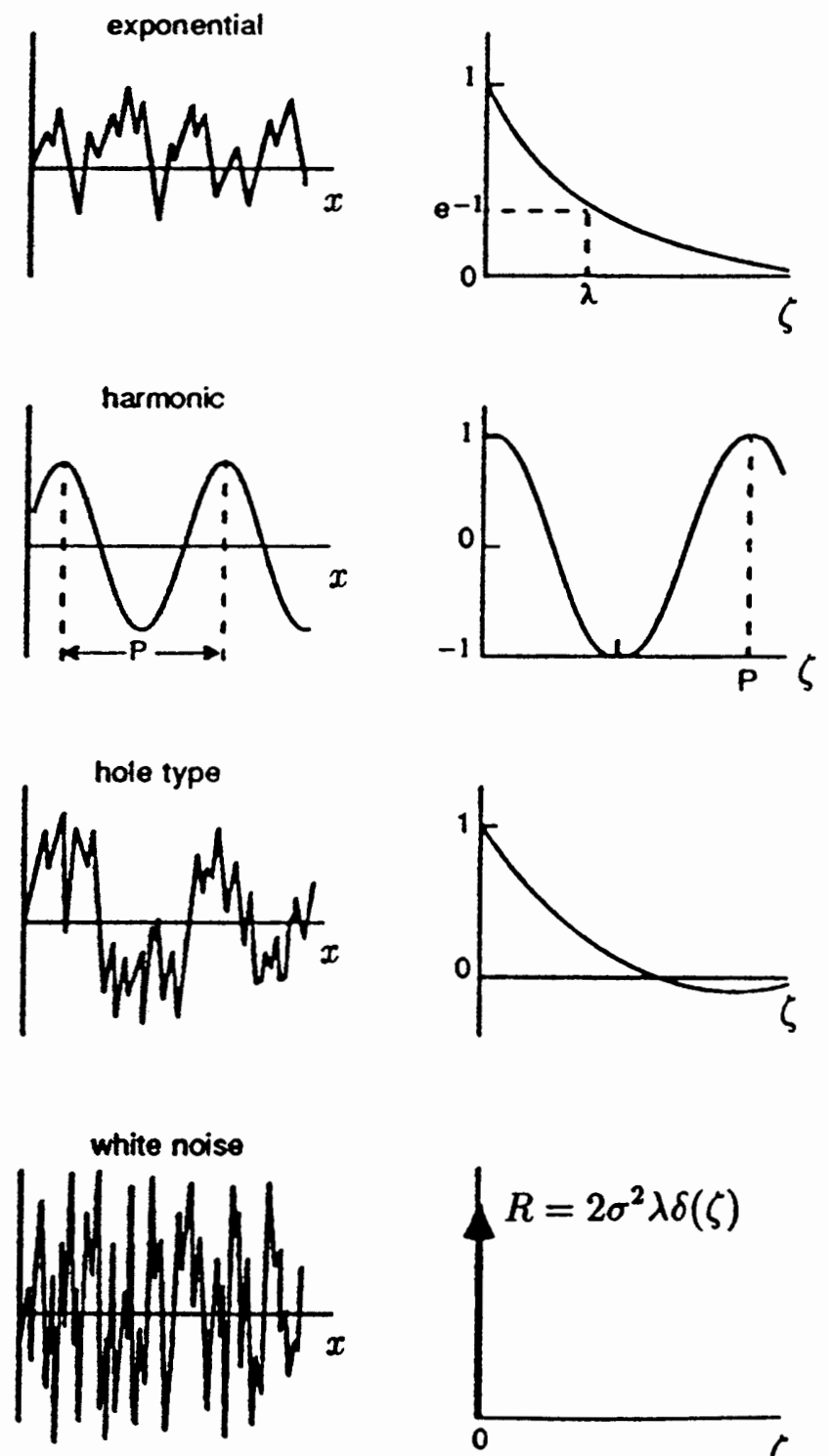

$R_{f f}(\zeta) / \sigma^{2}$
$S_{f f}(\omega)$
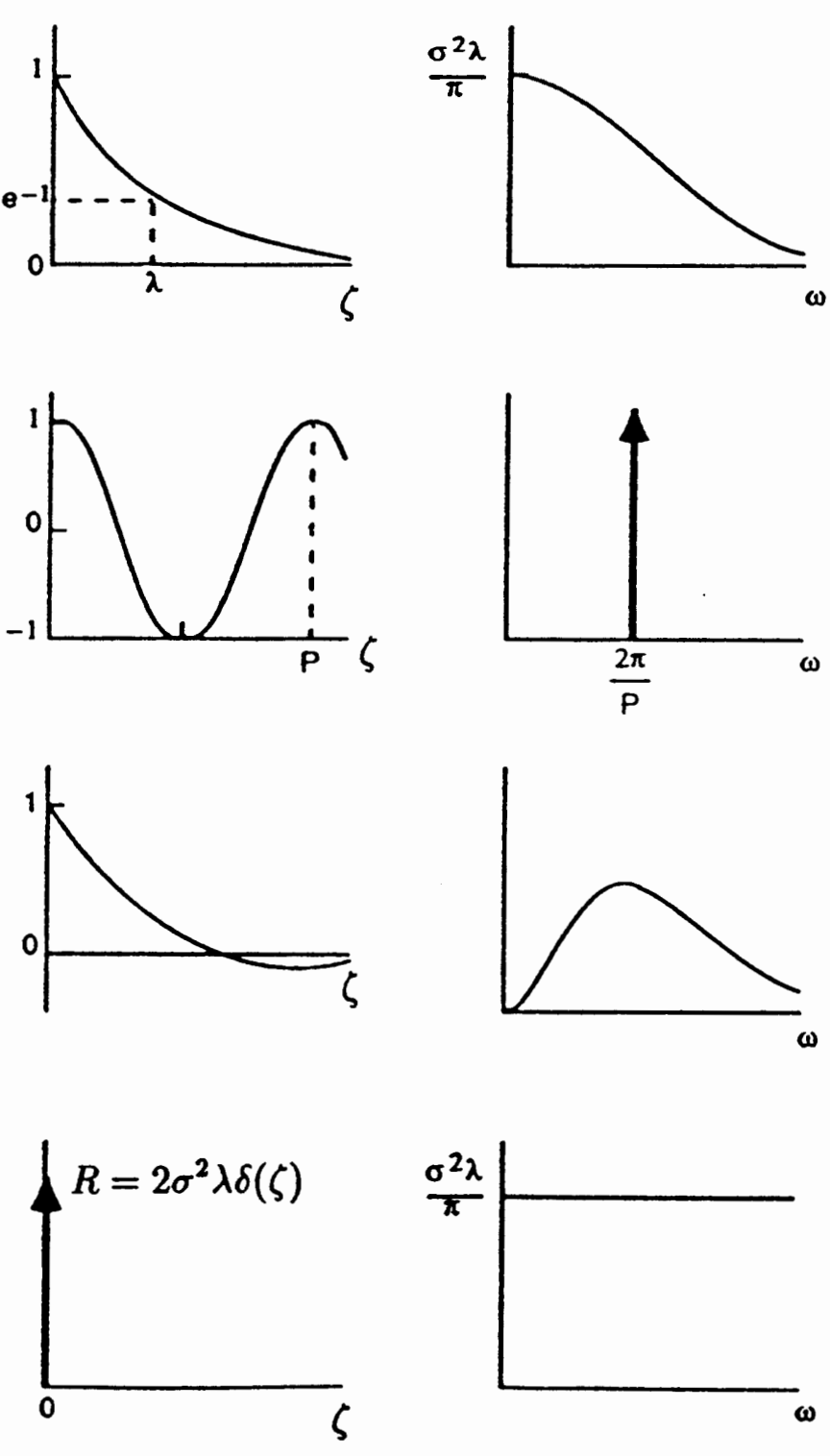

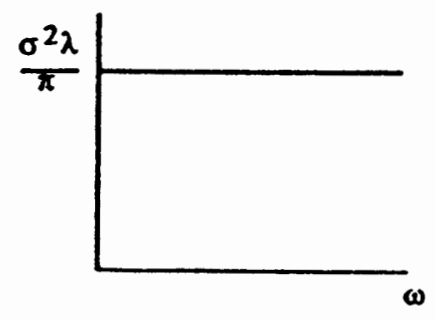

Figure 3-4: Schematic representation of different types of covariance-spectrum pairs. (Source: Gelhar, 1993, pp. 33) 
frequency the spectral amplitude reaches to zero. The "hole" type covariancespectrum pair can describe the stream geometrical variations relatively well. This is why the "hole" exponential function is used in the thesis.

\subsection{Stochastic Transport Analysis}

\subsubsection{Stochastic Solute Transport Equation}

The equation describing the longitudinal transport of an ideal conservative solute by a steady flow in a natural river is (Cunge et al, 1986)

$$
\frac{\partial c}{\partial t}+U(x) \frac{\partial c}{\partial x}=\frac{1}{A(x)} \frac{\partial}{\partial x}\left[A(x) K(x) \frac{\partial c}{\partial x}\right]
$$

which can be rearranged in the following standard advective diffusion equation with a modified flow velocity

$$
\frac{\partial c}{\partial t}+V(x) \frac{\partial c}{\partial x}=K(x) \frac{\partial^{2} c}{\partial x^{2}}
$$

where

$$
V(x)=U(x)-\frac{1}{A(x)} \frac{\partial}{\partial x}[A(x) K(x)]
$$

where $c$ is cross-sectional concentration of the transported solute, $U$ is crosssectional mean flow velocity, $t$ is time, $A$ is the cross-section area, and $K$ is the 
longitudinal dispersion coefficient accounting for flow variation within the flow cross-section and is given by (Fischer, 1967)

$$
\begin{aligned}
K(x)= & -\frac{1}{A(x)} \int_{y_{l}(x)}^{y_{r}(x)} U^{\prime \prime}(x, \mu) d(x, \mu) \int_{y_{l}(x)}^{\mu} \frac{1}{E_{z} d(x, \eta)} \\
& \int_{y_{l}(x)}^{\eta} U^{\eta}(x, \xi) d(x, \xi) d \xi d \eta d \mu
\end{aligned}
$$

where $y_{l}(x)$ and $y_{r}(x)$ are respectively the transverse coordinates of the left and right side of river bank, $d$ is the flow depth at any point, $U^{n}$ is the velocity deviation from its cross-sectional mean, $\mu, \eta$, and $\xi$ are dummy integration variables, and $E_{z}$ is the transverse dispersion coefficient.

Equation (3.9) is a stochastic partial differential equation since $V(x)$ and $K(x)$ are functions of random river geometric variables. The random quantities $c, V$ and $K$, can each be expanded into the sum of the mean (denoted by an overbar) and a small perturbation around this mean (denoted by a prime), or

$$
c=\bar{c}+c^{\prime} ; V=\bar{V}+V^{\prime} ; K=\bar{K}+K^{\prime}
$$

Substituting Eqn. (3.12) into Eqn. (3.9) yields:

$$
\frac{\partial\left(\bar{c}+c^{\prime}\right)}{\partial t}+\left(\bar{V}+V^{\prime}\right) \frac{\partial\left(\bar{c}+c^{\prime}\right)}{\partial x}=\left(\bar{K}+K^{\prime}\right) \frac{\partial^{2}\left(\bar{c}+c^{\prime}\right)}{\partial x^{2}}
$$


Expanding terms and averaging both sides produces the ensemble mean equation:

$$
\frac{\partial \bar{c}}{\partial t}+\bar{V} \frac{\partial \bar{c}}{\partial x}=\bar{K} \frac{\partial^{2} \bar{c}}{\partial x^{2}}-\overline{V^{\prime} \frac{\partial c^{\prime}}{\partial x}}+\overline{K^{\prime} \frac{\partial^{2} c^{\prime}}{\partial x^{2}}}
$$

where the last two terms on the right-hand side reflect the additional contribution to dispersive flux due to longitudinal random variations in $V$ and $K$. Evaluation of these dispersive fluxes induced by longitudinal flow nonuniformity is the focus of the rest of this analysis.

Subtracting Eqn. (3.14) from Eqn. (3.13) gives the perturbation equation

$$
\frac{\partial c^{\prime}}{\partial t}+\bar{V} \frac{\partial c^{\prime}}{\partial x}=\bar{K} \frac{\partial^{2} c^{\prime}}{\partial x^{2}}-V^{\prime} \frac{\partial \bar{c}}{\partial x}+K^{\prime} \frac{\partial^{2} \bar{c}}{\partial x^{2}}-V^{\prime} \frac{\partial c^{\prime}}{\partial x}+\overline{V^{\prime} \frac{\partial c^{\prime}}{\partial x}}+K^{\prime} \frac{\partial^{2} c^{\prime}}{\partial x^{2}}-\overline{K^{\prime} \frac{\partial^{2} c^{\prime}}{\partial x^{2}}}
$$

Adopting a moving coordinate system, $\xi=x-\bar{V} t$, assuming the perturbations $c^{\prime}, K^{\prime}$ and $V^{\prime}$ are small, and thus dropping the second order terms, I obtain the following first order approximation describing the concentration fluctuation

$$
\frac{\partial c^{\prime}}{\partial t}=\bar{K} \frac{\partial^{2} c^{\prime}}{\partial \xi^{2}}-V^{\prime} \frac{\partial \bar{c}}{\partial \xi}+K^{\prime} \frac{\partial^{2} \bar{c}}{\partial \xi^{2}}
$$

The equation governing the perturbation of concentration derivatives $G_{1}^{\prime}=\partial c^{\prime} / \partial \xi$ and $G_{2}^{\prime}=\partial^{2} c^{\prime} / \partial \xi^{2}=\partial G_{1}^{\prime} / \partial \xi$ can be obtained by differentiating Eqn. (3.16) with 
respect to $\xi$ as follows

$$
\frac{\partial G_{1}^{\prime}}{\partial t}=\bar{K} \frac{\partial^{2} G_{1}^{\prime}}{\partial \xi^{2}}-V^{\prime} \frac{\partial^{2} \bar{c}}{\partial \xi^{2}}-\frac{\partial V^{\prime}}{\partial \xi} \frac{\partial \bar{c}}{\partial \xi}+\frac{\partial K^{\prime}}{\partial \xi} \frac{\partial^{2} \bar{c}}{\partial \xi^{2}}
$$

where the terms containing derivatives higher than second order have been neglected, because I am only interested in the longitudinal dispersion coefficient which is related to the second spatial derivative of concentration. The following identity is used to derive Eqn. (3.17)

$$
\frac{\partial G_{1}^{\prime}}{\partial t}=\frac{\partial}{\partial t} \frac{\partial c^{\prime}}{\partial \xi}=\frac{\partial}{\partial \xi}\left(\frac{\partial c^{\prime}}{\partial t}\right)
$$

Equations (3.16) and (3.17) are the approximate stochastic differential equations describing the perturbation of concentration and its derivatives produced as a result of $V^{\prime}$ and $K^{\prime}$, which are in turn related to the fluctuations in river geometry. I next evaluate the ensemble mean of the modified flow velocity, $V$, and Fischer's dispersion coefficient, $K$, and their first order perturbations $V^{\prime}$ and $K^{\prime} . K(x)$ is given by the triple integral expression in Eqn. (3.11); it is related to flow and geometric variables in a complicated, nonlinear way. Evaluation of $\mathrm{x}$-variation of longitudinal dispersion $K(x)$ requires information on transverse distributions of flow depth and velocity at different locations $x$. If the mean transverse distributions of flow depth and velocity are denoted as, respectively, $h_{1}(y)$ and $U_{1}(y)$ (averaged along the flow), 


$$
\begin{aligned}
& h_{1}(y)=\frac{1}{L} \int_{0}^{L} d(x, y) d x \\
& U_{1}(y)=\frac{1}{L} \int_{0}^{L} U(x, y) d x
\end{aligned}
$$

where $L$ is the length of the longitudinal transport zone.

laterally averaged flow depth and velocity as $h(x)$ and $U(x)$, and the global mean flow depth and velocity as $\bar{h}$ and $\bar{U}$ (averaged throughout the flow both in $x$ and $y$ direction), the lateral distribution of flow depth and velocity at any location $x$ can be approximated as

$$
\begin{aligned}
d(x, y) & =\frac{h(x)}{\bar{h}} h_{1}(y) \\
U(x, y) & =\frac{U(x)}{\bar{U}} U_{1}(y)
\end{aligned}
$$

This approximation is necessary in order to obtain an explicit expression of $K$ and $U$ as a function of $x$. Substituting Eqns. (3.21) and (3.22) in Eqn. (3.11) and factoring out terms which are only functions of $x$ yields

$$
K(x)=\frac{U(x)^{2} h(x) \bar{A}}{\bar{U}^{2} \bar{h} A(x)} K_{0}
$$

where $K_{0}$ is the longitudinal dispersion coefficient for a uniform channel with mean geometry 


$$
K_{0}=-\frac{1}{\bar{A}} \int_{\bar{y}_{l}}^{\bar{y}_{r}} U_{1}{ }^{\eta}(\mu) h_{1}(\mu) \int_{\bar{y}_{l}}^{\mu} \frac{1}{E_{z} h_{1}(\eta)} \int_{\bar{y}_{l}}^{\eta} U_{1}{ }^{\eta}(\xi) h_{1}(\xi) d \xi d \eta d \mu
$$

where $U_{1}$ " is the deviation of $U(x, y)$ from cross-sectional mean velocity.

Note that the integration from $y_{l}$ and $y_{r}$ has been assumed to be approximately the same as integrating over the mean river width. The error committed in this approximation is of the order $U_{1}^{n}\left(\bar{y}_{r}\right) h\left(\bar{h}_{r}\right) \sigma_{B}$, which is considered negligible since variance of width and flow depth at the river bank are both very small.

Each random quantity in Eqn. (3.23) can be expanded into the sum of its mean and a small pertubation around this mean, or

$$
\begin{aligned}
& A=\bar{A}+A^{\prime} \\
& U=\bar{U}+U^{\prime} \\
& h=\bar{h}+h^{\prime}
\end{aligned}
$$

where the overbar denotes ensemble mean, which is also equivalent to the spatical mean along the longitudinal direction if the ergodicity or local ergodicity is invoked. In what follows, no difference is made between ensemble mean and longitudinal 
mean, these two mean are exchangable.

Substituting Eqns. (3.25) to (3.27) into Eqn. (3.23), expanding terms and taking an ensemble mean value on both sides of the equation; and assuming the perturbations $A^{\prime}, U^{\prime}$ and $h^{\prime}$ are small thus dropping the second order terms, also noting

$$
\frac{U^{\prime}(x)}{\bar{U}}=-\frac{A^{\prime}(x)}{\bar{A}}=-\left[\frac{h^{\prime}(x)}{\bar{h}}+\frac{B^{\prime}(x)}{\bar{B}}\right]
$$

yields

$$
\bar{K}=K_{0}\left(1+4 \frac{\sigma_{B}^{2}}{\bar{B}^{2}}+\frac{\sigma_{h}^{2}}{\bar{h}^{2}}+5 \frac{\sigma_{B h}}{\overline{B h}}\right)
$$

subtracting Eqn. (3.29) from Eqn.(3.23), obtains

$$
K^{\prime}=-K_{0}\left(2 \frac{h^{\prime}}{\bar{h}}+3 \frac{B^{\prime}}{\bar{B}}\right)
$$

Similarly, substituting each random quantity in Eqn.(3.10), expanding terms and taking a mean on both sides produces the longitudinal mean modified flow velocity $\bar{V}$

$$
\bar{V}=\bar{U}-\frac{\partial \bar{K}}{\partial x}-\frac{K^{\prime}}{\bar{A}} \frac{\partial A^{\prime}}{\partial x}
$$

A perturbation analysis gives 


$$
V^{\prime}(x)=U^{\prime}-\frac{\partial K^{\prime}}{\partial x}-\frac{\bar{K}}{\bar{A}} \frac{\partial A^{\prime}}{\partial x}
$$

Making use of Eqns. (3.28) through (3.30) into Eqns. (3.31) and (3.32), the following ensemble mean and perturbation of modified flow velocity equation can be obtained,

$$
\begin{gathered}
\bar{V}=\bar{U}-\frac{\partial \bar{K}}{\partial x}-\frac{K_{0} B^{\prime}}{\overline{B h}}\left(\frac{\partial h^{\prime}}{\partial x}\right) \\
V^{\prime}(x)=U^{\prime}+K_{0}\left(\frac{1}{\bar{h}} \frac{\partial h^{\prime}}{\partial x}+\frac{2}{\bar{B}} \frac{\partial B^{\prime}}{\partial x}\right)
\end{gathered}
$$

\subsubsection{Closed-Form Spectral Solutions}

By assuming local statistical homogeneity, the solutions of Eqn. (3.17) are developed using the Fourier-Stieltjes representations for the perturbation quantities $V^{\prime}$, $K^{\prime}, G_{1}^{\prime}, G_{2}^{\prime}$

$$
\begin{aligned}
& V^{\prime}=\int_{-\infty}^{\infty} e^{i \omega x} d Z_{V} \\
& K^{\prime}=\int_{-\infty}^{\infty} e^{i \omega x} d Z_{K} \\
& G_{1}^{\prime}=\int_{-\infty}^{\infty} e^{i \omega x} d Z_{G_{1}} \\
& G_{2}^{\prime}=\int_{-\infty}^{\infty} e^{i \omega x} d Z_{G_{2}}
\end{aligned}
$$


where the $d Z^{\prime} s$ are the complex Fourier amplitudes, $\omega$ is wave number. Invoking the uniqueness of the spectral representations (Priestley, 1981; Li et al., 1992), I obtain the following equation for the Fourier amplitude $d Z_{G_{1}}$

$$
\left(\frac{\partial}{\partial t}+\bar{K} \omega^{2}\right) d Z_{G_{1}}=-\left(i \omega \frac{\partial \bar{c}}{\partial \xi}+\frac{\partial^{2} \bar{c}}{\partial \xi^{2}}\right) d Z_{V}+i \omega \frac{\partial^{2} \bar{c}}{\partial \xi^{2}} d Z_{K}
$$

since $G_{2}=\partial G_{1}^{\prime} / \partial \xi, d Z_{G_{2}}=i \omega d Z_{G_{1}}$, the complex Fourier amplitude $d Z_{G_{2}}$ satisfies the following similar equation

$$
\left(\frac{\partial}{\partial t}+\bar{K} \omega^{2}\right) d Z_{G_{2}}=\left(\omega^{2} \frac{\partial \bar{c}}{\partial \xi}-i \omega \frac{\partial^{2} \bar{c}}{\partial \xi^{2}}\right) d Z_{V}-\omega^{2} \frac{\partial^{2} \bar{c}}{\partial \xi^{2}} d Z_{K}
$$

Multiplying both sides of Eqn. (3.39) by $d Z_{V}^{c}$, the complex conjugate of the Fourier amplitude $d Z_{V}$, and both sides of Eqn. (3.40) by $d Z_{K}^{c}$, taking the mean value, and noting the orthogonality between the Fourier amplitudes at different frequency, results in the following spectral relationships

$$
\begin{gathered}
\left(\frac{\partial}{\partial t}+\bar{K} \omega^{2}\right) S_{V G_{1}}=\left(i \omega \frac{\partial \bar{c}}{\partial \xi}-\frac{\partial^{2} \bar{c}}{\partial \xi^{2}}\right) S_{V V}-i \omega \frac{\partial^{2} \bar{c}}{\partial \xi^{2}} S_{V K} \\
\left(\frac{\partial}{\partial t}+\bar{K} \omega^{2}\right) S_{K G_{2}}=\left(\omega^{2} \frac{\partial \bar{c}}{\partial \xi}+i \omega \frac{\partial^{2} \bar{c}}{\partial \xi^{2}}\right) S_{K V}-\omega^{2} \frac{\partial^{2} \bar{c}}{\partial \xi^{2}} S_{K K}
\end{gathered}
$$

Note that Eqns. (3.41) and (3.42) are differential equations in time only, the spectral coordinate appears only parametrically through the derivatives of the mean concentration. Solving the two first order, linear, constant coefficient dif- 
ferential equations for $S_{V G_{1}}$ and $S_{K G_{2}}$ and integrating the resulting cross-spectra over the wave number domain yields

$$
\begin{gathered}
\overline{-V^{\prime} \frac{\partial c^{\prime}}{\partial \xi}}=\int_{-\infty}^{\infty} \int_{0}^{t} e^{\bar{K} \omega^{2}(\tau-t)}\left[S_{V V}+i \omega S_{V K}\right] \frac{\partial^{2} \bar{c}}{\partial \xi^{2}} d \tau d \omega \\
\overline{K^{\prime} \frac{\partial^{2} c^{\prime}}{\partial \xi^{2}}}=\int_{-\infty}^{\infty} \int_{0}^{t} e^{\bar{K} \omega^{2}(\tau-t)}\left[\omega^{2} S_{K V} \frac{\partial \bar{c}}{\partial \xi}+\left(i \omega S_{K V}-\omega^{2} S_{K K}\right) \frac{\partial^{2} \bar{c}}{\partial \xi^{2}}\right] d \tau d \omega
\end{gathered}
$$

where homogeneous initial conditions at $t=0$ have been assumed for $\partial c^{\prime} / \partial \xi$ and $\partial^{2} c^{\prime} / \partial \xi^{2}$. Eqns. (3.43) and (3.44) show that the additional dispersive fluxes due to $V^{\prime}$ and $K^{\prime}$ that show up in Eqn. (3.14) are not in general proportional to the local mean concentration gradients; rather, they are influenced by exponentially weighted contributions from earlier concentration gradients. The main contribution to the time integral in Eqns. (3.43) and (3.44) will be near the upper limit $\tau=t$. Therefore $\partial \bar{c} / \partial \xi$ and $\partial^{2} \bar{c} / \partial \xi^{2}$ are expanded as follows using a Taylor series

$$
\begin{gathered}
\frac{\partial \bar{c}(\xi, \tau)}{\partial \xi}=\frac{\partial \bar{c}(\xi, t)}{\partial \xi}+\frac{\partial^{2} \bar{c}(\xi, t)}{\partial \tau \partial \xi}(\tau-t)+\ldots \\
\frac{\partial^{2} \bar{c}(\xi, \tau)}{\partial \xi^{2}}=\frac{\partial^{2} \bar{c}(\xi, t)}{\partial \xi^{2}}+\frac{\partial^{3} \bar{c}(\xi, t)}{\partial \tau \partial \xi^{2}}(\tau-t)+\ldots
\end{gathered}
$$

Retaining only the first terms in these expansions, Eqns. (3.43) and (3.44) become

$$
\overline{-V^{\prime} \frac{\partial c^{\prime}}{\partial \xi}}=K_{V} \frac{\partial^{2} \bar{c}}{\partial \xi^{2}}
$$




$$
\overline{K^{\prime} \frac{\partial^{2} c^{\prime}}{\partial \xi^{2}}}=V_{K} \frac{\partial \bar{c}}{\partial \xi}+K_{K} \frac{\partial^{2} \bar{c}}{\partial \xi^{2}}
$$

where

$$
\begin{gathered}
K_{V}=\int_{-\infty}^{\infty} \frac{1-e^{-\bar{K} \omega^{2} t}}{\bar{K} \omega^{2}}\left[S_{V V}(\omega)+i \omega S_{V K}\right] d \omega \\
K_{K}=\int_{-\infty}^{\infty} \frac{1-e^{-\bar{K} \omega^{2} t}}{\bar{K} \omega^{2}}\left[i \omega S_{K V}-\omega^{2} S_{K K}(\omega)\right] d \omega \\
V_{K}=\int_{-\infty}^{\infty} \frac{1-e^{-\bar{K} \omega^{2} t}}{\bar{K}} S_{K V} d \omega
\end{gathered}
$$

As time becomes sufficiently large, Eqns. (3.49) through (3.51) become

$$
\begin{gathered}
K_{V}=\int_{-\infty}^{\infty} \frac{S_{V V}(\omega)+i \omega S_{V K}}{\bar{K} \omega^{2}} d \omega \\
K_{K}=\int_{-\infty}^{\infty} \frac{i \omega S_{K V}-\omega^{2} S_{K K}(\omega)}{\bar{K} \omega^{2}} d \omega \\
V_{K}=\int_{-\infty}^{\infty} \frac{S_{K V}}{\bar{K}} d \omega=\frac{\sigma_{K V}}{\bar{K}}
\end{gathered}
$$

Making use of Eqns. (3.30) and (3.34), I can relate the spectra $S_{V V}, S_{K K}$ and $S_{K V}$ in the above equations to those of velocity $U$, flow depth $h$ and river width $B$,

$$
\begin{gathered}
S_{V V}=\bar{U}^{2} S_{U U}-K_{0} i \omega \bar{U}\left(S_{B h}-S_{h B}\right)+K_{0}^{2} \omega^{2}\left(4 S_{B B}+S_{h h}+2 S_{B h}+2 S_{h B}\right) \\
S_{K K}=K_{0}^{2}\left(4 S_{U U}+5 S_{B B}+2 S_{B h}+2 S_{h B}\right)
\end{gathered}
$$


$S_{K V}=K_{0} \bar{U}\left(2 S_{h h}+3 S_{B B}+3 S_{B h}+2 S_{h B)}+K_{0}^{2} i \omega \bar{U}\left(2 S_{h h}+4 S_{h B}+6 S_{B B}+3 S_{B h}\right)\right.$

where $S_{h h}, S_{U U}$, and $S_{B h}$ are respectively the spectra of flow depth, velocity, and cross spectrum of river width and flow depth. These flow related spectra can be related to those of geometric variations by solving a gradually varying steady-state flow problem. This has already been done and reported in $\mathrm{Li}$ et al. (1992). The following is the summary of the relevant results,

$$
\begin{gathered}
S_{h h}=\frac{\bar{B}^{2} S_{z z} \omega^{2}+\bar{h}^{2} S_{B B}\left(4 s^{2}+F_{r}^{4} \omega^{2}\right)}{\left[9 s^{2}+\omega^{2}\left(1-F_{\tau}^{2}\right)^{2}\right] \bar{B}^{2} \bar{h}^{2}} \\
S_{U U}=\frac{\bar{B}^{2} S_{z z} \omega^{2}+\bar{h}^{2} S_{B B}\left(s^{2}+\omega^{2}\right)}{\left[9 s^{2}+\omega^{2}\left(1-F_{r}^{2}\right)^{2}\right] \bar{B}^{2} \bar{h}^{2}} \\
S_{B h}=\frac{S_{B B}\left(-6 s^{2}+2 i s \omega+i s \omega F_{\tau}^{2}+F_{r}^{2} \omega^{2}-F_{\tau}^{4} \omega^{2}\right)}{\bar{B}^{2}\left[9 s^{2}+\omega^{2}\left(1-F_{r}^{2}\right)^{2}\right]}
\end{gathered}
$$

where $F r$ is ensemble mean flow Froude number, defined as $F r=\bar{U} / \sqrt{g \bar{h}}, s$ is river bed slope, $S_{B B}$ and $S_{z z}$ are the power spectra of river width and bed elevation, respectively.

\subsubsection{Effective Mean Transport Equation}

Substituting Eqns. (3.47) and (3.48) in Eqn. (3.14), the following equation is obtained which describes the large scale mean behavior of longitudinal solute trans- 
port in a natural stream,

$$
\frac{\partial \bar{c}}{\partial t}+V_{e} \frac{\partial \bar{c}}{\partial x}=K_{e} \frac{\partial^{2} \bar{c}}{\partial x^{2}}
$$

where

$$
\begin{gathered}
V_{e}=\bar{V}-V_{K} \\
K_{e}=\bar{K}+K_{V}+K_{K}
\end{gathered}
$$

Equation (3.61) is of the same form as that governing solute transport in a uniform channel. This equation will be referred to as effective mean solute transport equation. Evaluation of the effective transport properties, effective longitudinal dispersion coefficient, $K_{e}$, and the effective mean velocity, $V_{e}$, are the focus of rest of the thesis.

At this point, the functional form of the covariance for the random geometric variations are needed before an explicit expression for the effective parameters can be obtained. As I point out before, the hole-exponential covariance function given in Eqn. (3.6) well describes variation in both river width and river bed elevation. Their corresponding spectra are, replacing $f$, respectively, with the symbol $B, z$, representing river width and bed elevation in Eqn. (3.7),

$$
S_{B B}=\frac{4 \sigma_{B}^{2}}{3 \pi^{2}} \frac{\omega^{3} \lambda_{B}^{3}}{\left(1+\omega^{2} \lambda_{B}^{2}\right)^{3}}
$$




$$
S_{z z}=\frac{4 \sigma_{z}^{2}}{3 \pi^{2}} \frac{\omega^{3} \lambda_{z}^{3}}{\left(1+\omega^{2} \lambda_{z}^{2}\right)^{3}}
$$

where $S_{B B}, S_{z z}$ are the power spectra of river width and bed elevation; $\sigma_{B}^{2}$, and $\sigma_{z}^{2}$ are variances of river width and bed elevation; $\lambda_{B}$ and $\lambda_{z}$ are the correlation scales of river width and bed elevation, respectively.

Substituting Eqns. (3.64) and (3.65) into Eqns.(3.58) through (3.60), which are in turn substituted into Eqns. (3.55) through (3.57). Finally Eqns.(3.55) through (3.57) are substituted into Eqns. (3.52) to (3.54). Noting $S_{K V}=S_{V K}^{c}$, and carrying out the integration analytically, I have

$$
\frac{K_{e}}{K_{0}}=1+\alpha_{1} \frac{\sigma_{B}^{2}}{\bar{B}^{2}}+\alpha_{2} \frac{\sigma_{z}^{2}}{\bar{h}^{2}}
$$

where $\alpha_{1}$ and $\alpha_{2}$ are functions of mean Froude number $F_{r}$, and four other mean flow related dimensionless parameters: $P_{B}\left(=\lambda_{B} \bar{U} / K_{0}\right)$ or $P_{z}\left(=\lambda_{Z} \bar{U} / K_{0}\right), \Gamma_{B}(=$ $\left.\lambda_{B} s / \bar{h}\right)$ or $\Gamma_{z}\left(=\lambda_{z} s / \bar{h}\right)$. The final expressions for $\alpha_{1}$ and $\alpha_{2}$ are

$$
\begin{aligned}
\alpha_{1}= & \frac{1}{\pi \theta_{1}^{3}}\left\{4+\frac{1}{18}\left[9 \theta_{0}\left(3 \Gamma_{B}^{2}+1\right)+8 \Gamma_{B}\left(F_{r}-1\right)^{2}\right] P_{B}^{2}\right\} \\
& +\frac{1}{\pi \theta_{1}^{3}}\left\{\left(F_{r}^{2}+2\right) \theta_{0} \Gamma_{B} P_{B}+\frac{1}{6}\left[\theta_{2}\left(7 F_{r}^{2}-4 F_{r}^{4}-5\right)-105 \Gamma_{B}^{2} \theta_{0}\right]\right\}
\end{aligned}
$$




$$
\alpha_{2}=\frac{1}{6 \pi \psi_{1}^{3}}\left[3 P_{z}^{2} \psi_{0}-2 \psi_{2}\right]
$$

where

$$
\begin{aligned}
& \theta_{i}=(3)^{i} \Gamma_{B}+1-F_{r}^{2} \quad(i=0,1,2) \\
& \psi_{i}=(3)^{i} \Gamma_{z}+1-F_{r}^{2} \quad(i=0,1,2)
\end{aligned}
$$

Similarly, by evaluating Eqn. (3.54) and substituting the result into Eqn. (3.62), after some manipulation the result for the effective solute transport velocity is

$$
\frac{V_{e}}{\bar{U}}=1-\beta_{1} \frac{\sigma_{B}^{2}}{\bar{B}}-\beta_{2} \frac{\sigma_{z}^{2}}{\bar{h}}
$$

where $\beta_{1}$ and $\beta_{2}$ are functions of Froude number $F_{r}, P_{B}\left(=\lambda_{B} \bar{U} / K_{0}\right)$ or $P_{2}(=$ $\left.\lambda_{Z} \bar{U} / K_{0}\right)$, and $\Gamma_{B}\left(=\lambda_{B} s / \bar{h}\right)$ or $\Gamma_{z}\left(=\lambda_{z} s / \bar{h}\right)$. The final expressions for $\beta_{1}$ and $\beta_{2}$ are

$$
\begin{gathered}
\beta_{1}=\frac{1}{6 \pi \theta_{1}^{3}}\left\{\frac{1}{P_{B}}\left(F_{\tau}^{2}+2\right) \theta_{2} \Gamma_{B}+\left[F_{r}^{4}-4 F_{r}^{2}+15 \Gamma_{B}^{2} \theta_{0}+3\right]\right\} \\
+\frac{1}{6 \pi \theta_{1}^{3}}\left\{\left[9 \Gamma_{B}\left(3-F_{r}^{2}\right)+\frac{\Gamma_{B}}{P_{B}}\left(2-F_{r}^{4}-F_{r}^{2}\right) \frac{\Gamma_{B}^{3}}{P_{B}^{2}}\left(3 F_{\tau}^{2}-6\right)\right]\right\} \\
\beta_{2}=\frac{\psi_{2}}{3 \pi \psi_{1}^{3}}
\end{gathered}
$$


where $\theta_{i}$ and $\psi_{i}$ are as defined previously.

To summarize, an effective value for the mean longitudinal dispersion coefficient, $K_{e}$, has been found in terms of the previously defined uniform channel coefficient, $K_{0}$, and additional terms that account for variabilities in real streams

$$
K_{e}=\left(1+\alpha_{1} \frac{\sigma_{B}^{2}}{\bar{B}^{2}}+\alpha_{2} \frac{\sigma_{z}^{2}}{\bar{h}^{2}}\right) K_{0}
$$

I also have developed an expression for an effective solute transport velocity, $V_{e}$, based on the mean flow in a uniform channel, $\bar{U}$, and additional terms that account for variabilities

$$
V_{e}=\left(1-\beta_{1} \frac{\sigma_{B}^{2}}{\bar{B}}-\beta_{2} \frac{\sigma_{z}^{2}}{\bar{h}}\right) \bar{U}
$$

It is interesting to point out that the above two expressions indicate that the effective longitudinal dispersion coefficient, $K_{e}$, is always greater than that in uniform channel coefficient, $K_{0}$. However, the effective solute transport velocity, $V_{e}$, is always smaller than the mean flow velocity in a uniform channel, $\bar{U}$. This is because the parameters value of $\alpha_{1}, \alpha_{2}, \beta_{1}, \beta_{2}$ are all positive. The next chapter will further discuss and simplify the results. 


\section{Chapter 4}

\section{Discussion and Evaluation of}

\section{Results}

I have demonstrated that large-scale mean solute transport in irregular, but statistically uniform, streams can be described by the classical convective dispersion equation, as shown in Eqn. (3.61).

$$
\frac{\partial \bar{c}}{\partial t}+V_{e} \frac{\partial \bar{c}}{\partial x}=K_{e} \frac{\partial^{2} \bar{c}}{\partial x^{2}}
$$

This is the same equation governing solute transport in uniform channel but based on a modified or effective solute transport velocity and an effective longitudinal dispersion coefficient. These effective transport properties, as shown in Eqn. (3.74) and Eqn. (3.75), 


$$
\begin{gathered}
K_{e}=\left(1+\alpha_{1} \frac{\sigma_{B}^{2}}{\bar{B}^{2}}+\alpha_{2} \frac{\sigma_{z}^{2}}{\bar{h}^{2}}\right) K_{0} \\
V_{e}=\left(1-\beta_{1} \frac{\sigma_{B}^{2}}{\bar{B}}-\beta_{2} \frac{\sigma_{z}^{2}}{\bar{h}}\right) \bar{U}
\end{gathered}
$$

are strongly influenced by river cross-sectional nonuniformity, characterized by the statistical parameters $\sigma_{B}, \sigma_{z}, \lambda_{B}$, and $\lambda_{z}$, as well as by mean flow conditions, characterized by the mean Froude number and other flow related dimensionless groupings. To examine how different factors affect mean behavior of solute transport and dispersion in natural rivers, I have plotted the normalized effective transport properties, $K_{e} / K_{0}$ and $V_{e} / \bar{U}$, versus different characteristic parameters, including variance of river width and bed elevation, the Froude number and other mean flow related dimensionless groupings. The calculations are based on a set of nominal parameters shown in Table 4.1. The results are summarized in Figures (4-1) through (4-10) as presented in the next two subsections.

\subsection{Effective Dispersion Coefficient}

Figure (4-1) shows contours of the normalized effective longitudinal dispersion coefficient, $K_{\mathrm{e}} / K_{0}$, as a function of coefficient of variation in river width and bed elevation for the nominal case. It can be seen, the effective dispersion coefficient is always greater than the corresponding uniform mean channel dispersion coef- 


\begin{tabular}{|c|c|c|c|c|c|}
\hline Figure Number & $F_{r}$ & $\begin{array}{c}\frac{\lambda_{B^{s}}}{B} \\
\left(\Gamma_{B}\right)\end{array}$ & $\begin{array}{c}\frac{\lambda_{z^{8}}}{h} \\
\left(\Gamma_{z}\right)\end{array}$ & $\begin{array}{l}\frac{\lambda_{B} \bar{U}}{K_{0}} \\
\left(P_{B}\right)\end{array}$ & $\begin{array}{l}\frac{\lambda_{z} \bar{U}}{K_{0}} \\
\left(P_{z}\right)\end{array}$ \\
\hline $4-1$ & 0.4 & 0.001 & 0.001 & 1 & 1 \\
\hline $4-2$ & - & 0.001 & 0.001 & - & \\
\hline $4-3$ & $\begin{array}{l}0.2 \\
0.4 \\
0.6\end{array}$ & $\cdots$ & & 1 & \\
\hline $4-4$ & 0.4 & $\cdots$ & & $\begin{array}{l}0.2 \\
0.4 \\
0.6\end{array}$ & \\
\hline $4-5$ & 0.2 & 0.001 & 0.001 & 1 & 1 \\
\hline $4-6$ & 0.4 & 0.001 & 0.001 & 1 & 1 \\
\hline $4-7$ & 0.6 & 0.001 & 0.001 & 1 & 1 \\
\hline $4-8$ & $\ldots$ & 0.001 & 0.001 & 1 & 1 \\
\hline $4-9$ & 0.4 & 0.001 & 0.001 & - & $\cdots$ \\
\hline $4-10$ & 0.4 & $\ldots$ & - & 1 & 1 \\
\hline
\end{tabular}

Table 4.1: Parameter values used in Figures 4-1 through 4-10 


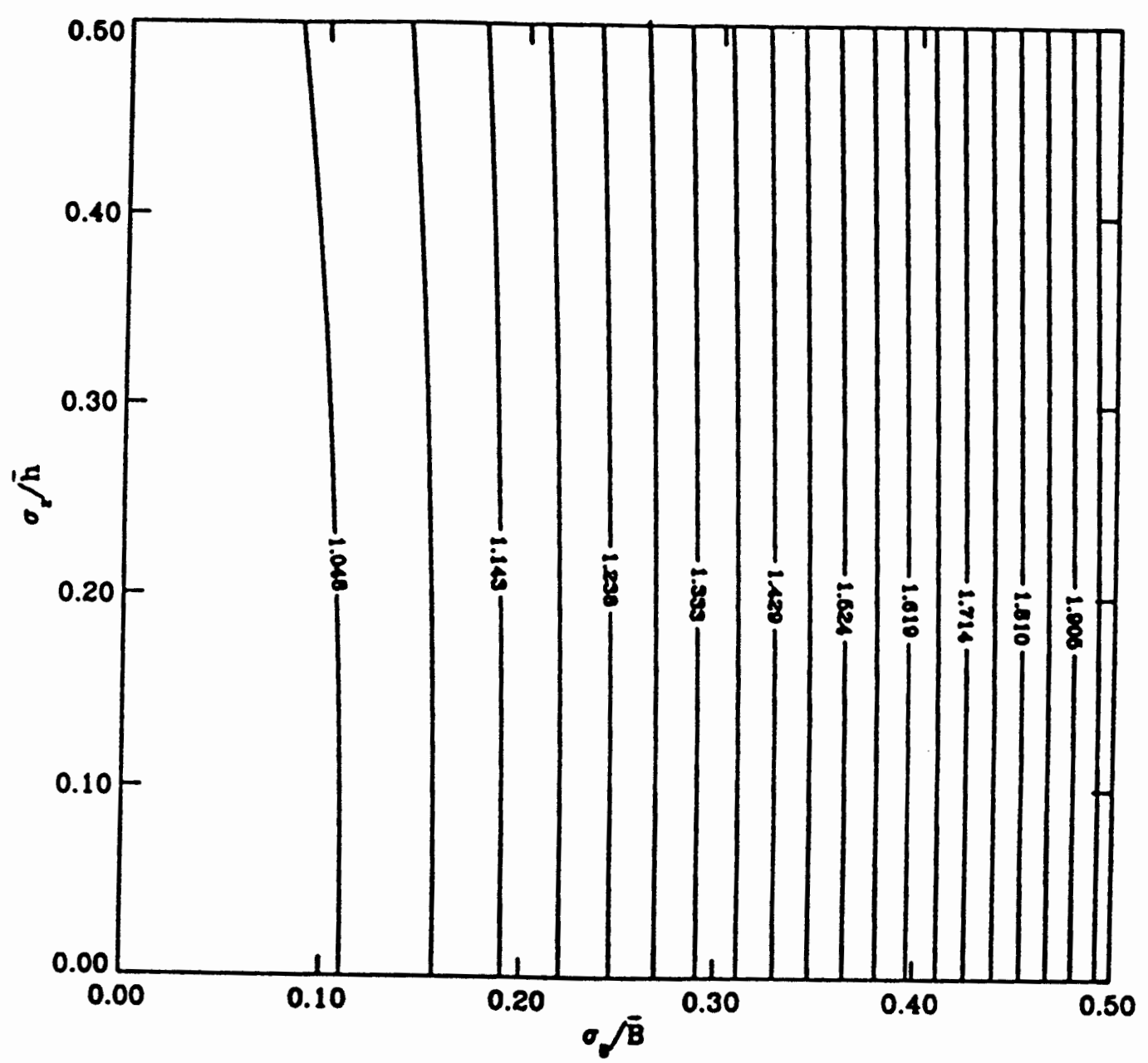

Figure 4-1: Contours of normalized effective longitudinal dispersion coefficient versus the coefficient of variation of river width and bed elevation 
ficient due to river cross-sectional nonuniformity. Effective dispersion coefficient increases quadratically with geometric nonuniformity, as measured by $\sigma_{B} / \bar{B}$ and $\sigma_{z} / \bar{h}$. But the sensitivity of the effective dispersion coefficient to $\sigma_{B} / \bar{B}$ and $\sigma_{z} / \bar{h}$ is dramatically different. Figure (4-1) clearly shows that dispersion coefficient is virtually independent of $\sigma_{z} / \bar{h}$. This insensitivity to river bed elevation also holds when the effective dispersion coefficient is evaluated for other mean flow related parameters. For all practical purposes the influence of variability in bed elevation on solute dispersion can be ignored. This is because, according to Taylor's theory, the longitudinal dispersion coefficient is proportional to the square of the width to depth ratio. A natural stream usually has a width to depth ratio in the range of ten or greater (Fischer et al., 1979). Thus the variation of river width is usually considerably more important in producing longitudinal dispersion than the variation of river bed elevation. So Eqn. (3.74) can be simplified

$$
K_{e}=\left(1+\alpha_{1} \frac{\sigma_{B}^{2}}{\bar{B}^{2}}\right) K_{0}
$$

An important characteristic of solute dispersion in natural streams is the mean flow dependence of the effective dispersion coefficient. The relative discrepancy between effective dispersion coefficient and the corresponding uniform channel dispersion coefficient is reflected by the magnitude of $\alpha_{1}$, or $\left(K_{e} / K_{0}-1\right) /\left(\sigma_{B}^{2} / \bar{B}^{2}\right)$. Figure (4-2) shows how $\alpha_{1}$ is influenced by the mean Froude number and $P_{B}\left(=\bar{U} \lambda_{B} / K_{0}\right)$. As I expected, $\alpha_{1}$ increases as $F_{r}$ and $P_{B}$ increases, but its sensitivity to $F_{r}$ and $P_{B}$ 


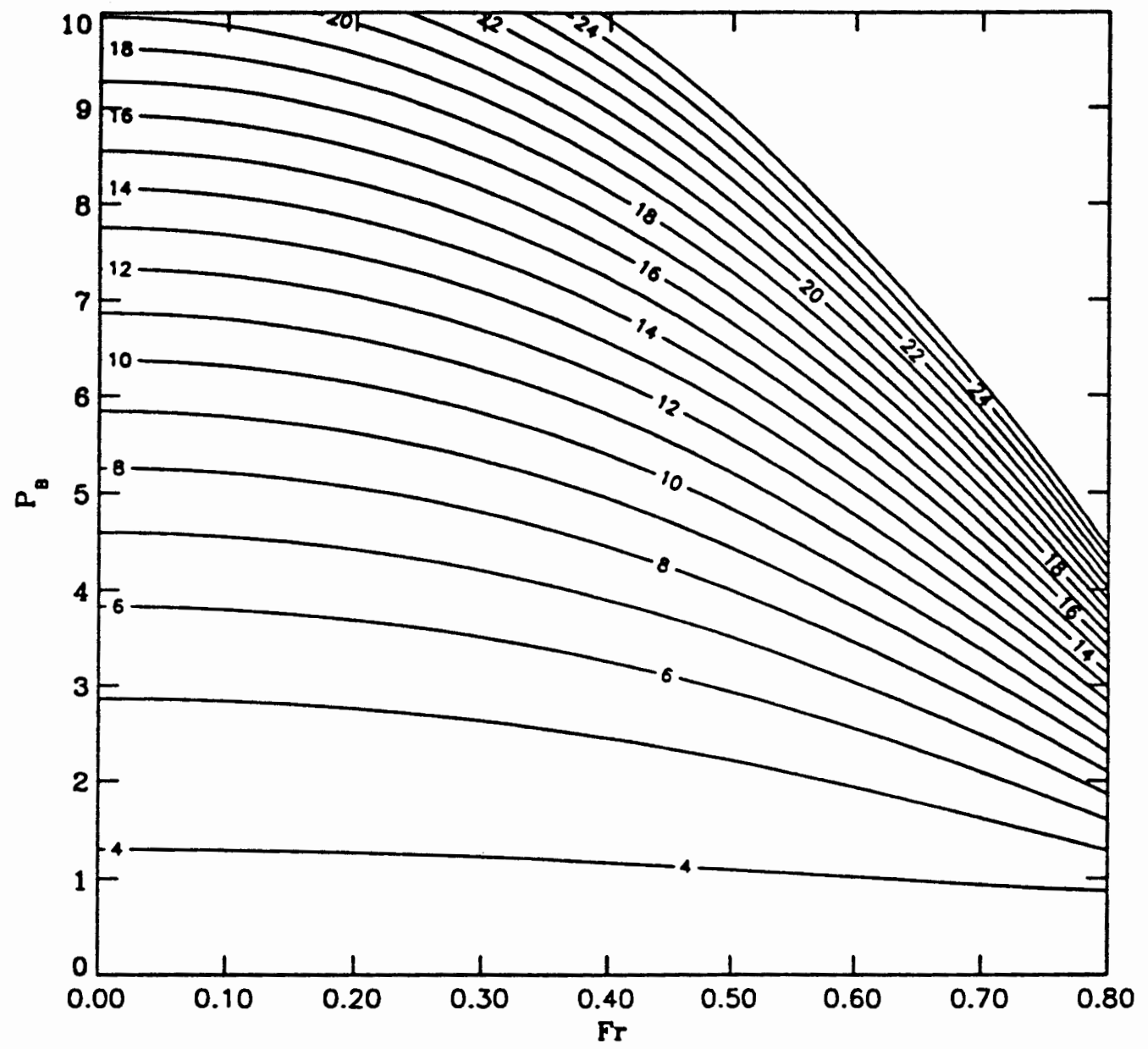

Figure 4-2: Contours of coefficient $\alpha_{1}$ versus the Froude number and $\bar{U} \lambda_{B} / K_{0}$ (denoted as $P_{B}$ ) 
is somewhat different. The coefficient $\alpha_{1}$ increases faster with $P_{B}$, especially when $F_{\tau}$ is high. This reflects the fact that flows in natural rivers become increasingly sensitive to boundary variations and that boundary nonuniformity induced dispersion becomes more and more important with increasing Froude number. Note that the effective longitudinal dispersion coefficient becomes unbounded as the Froude number approaches unity. This resonant behavior corresponds to the physical flow transition from subcritical state to a supercritical state. In fact, the results derived do not apply when $F_{\tau}$ approaches one or when $P_{B}$ is too large since in this case head and velocity variance is too large and the basic assumption of small perturbation is invalid.

Figure (4-3) and (4-4) show the relationship between $\alpha_{1}$ and $\Gamma_{B}$ for nominal values of $F_{\tau}$ and $P_{B}$. As I can see, $\alpha_{1}$ does not seem to vary with $\Gamma_{B}$. Evaluation of $\alpha_{1}$ for other sensitivity values of $F_{r}$ and $P_{B}$ shows the same trend. This suggests that I can further simply the expression for calculating $\alpha_{1}$, by ignoring $\Gamma_{B}$ in Eqn. (3.67). In so doing, $\alpha_{1}$ reduces to a function of only two dimensionless parameters, $P_{B}$, and the Froude number, $F_{r}$,

$$
\alpha_{1}=\frac{8+\left(1-F_{r}^{2}\right) P_{B}^{2}}{2 \pi\left(1-F_{r}^{2}\right)^{3}}
$$

This mean flow dependence of $\alpha_{1}$ explains why the predictions by existing empirical and semi-empirical theories when applied to the same rivers may agree with the observation in one instance but miss considerably in another due to changing 


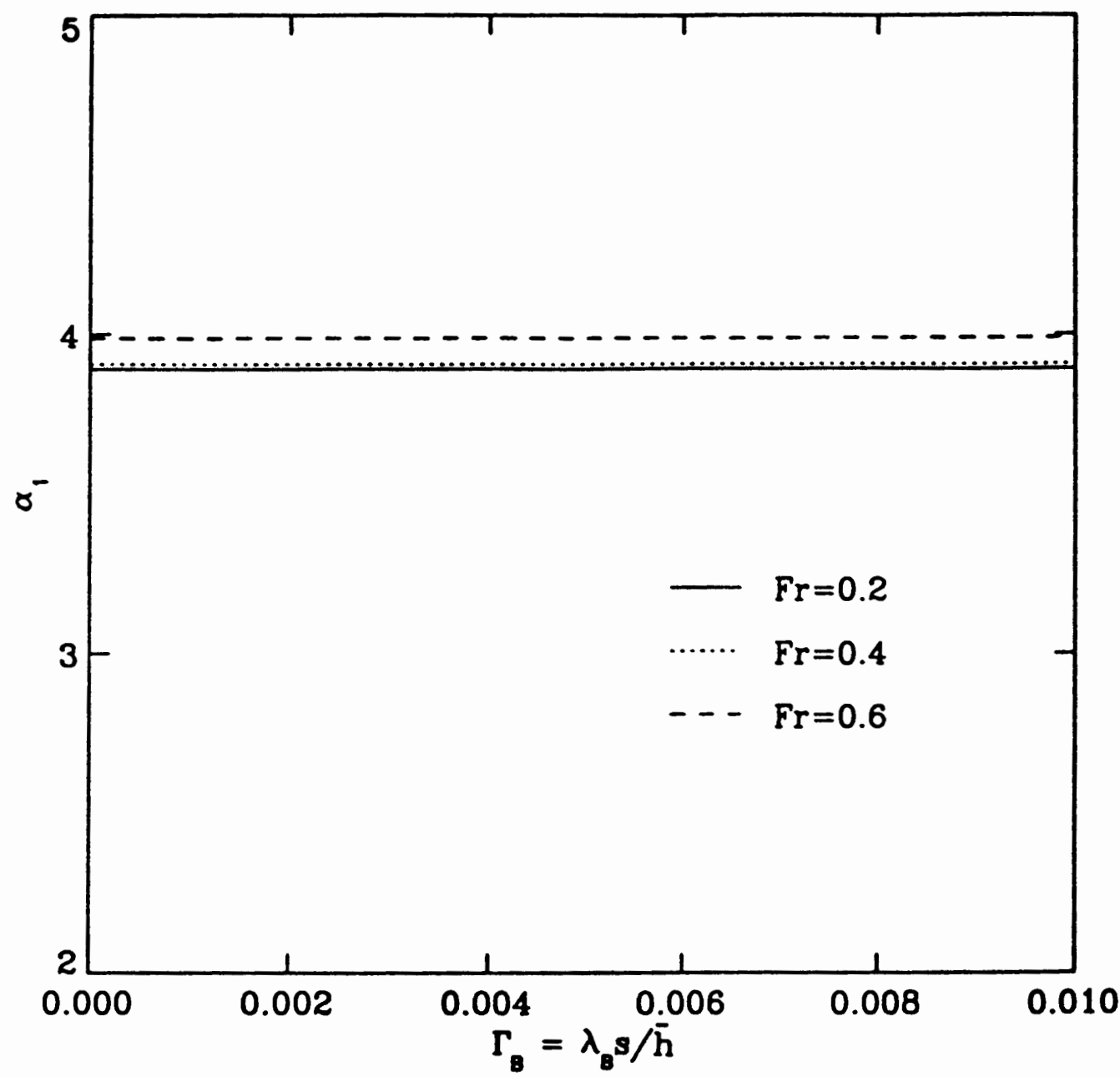

Figure 4-3: Effect of $\Gamma_{B}$ on the coefficient $\alpha_{1}$ for different $F_{\tau}$ 


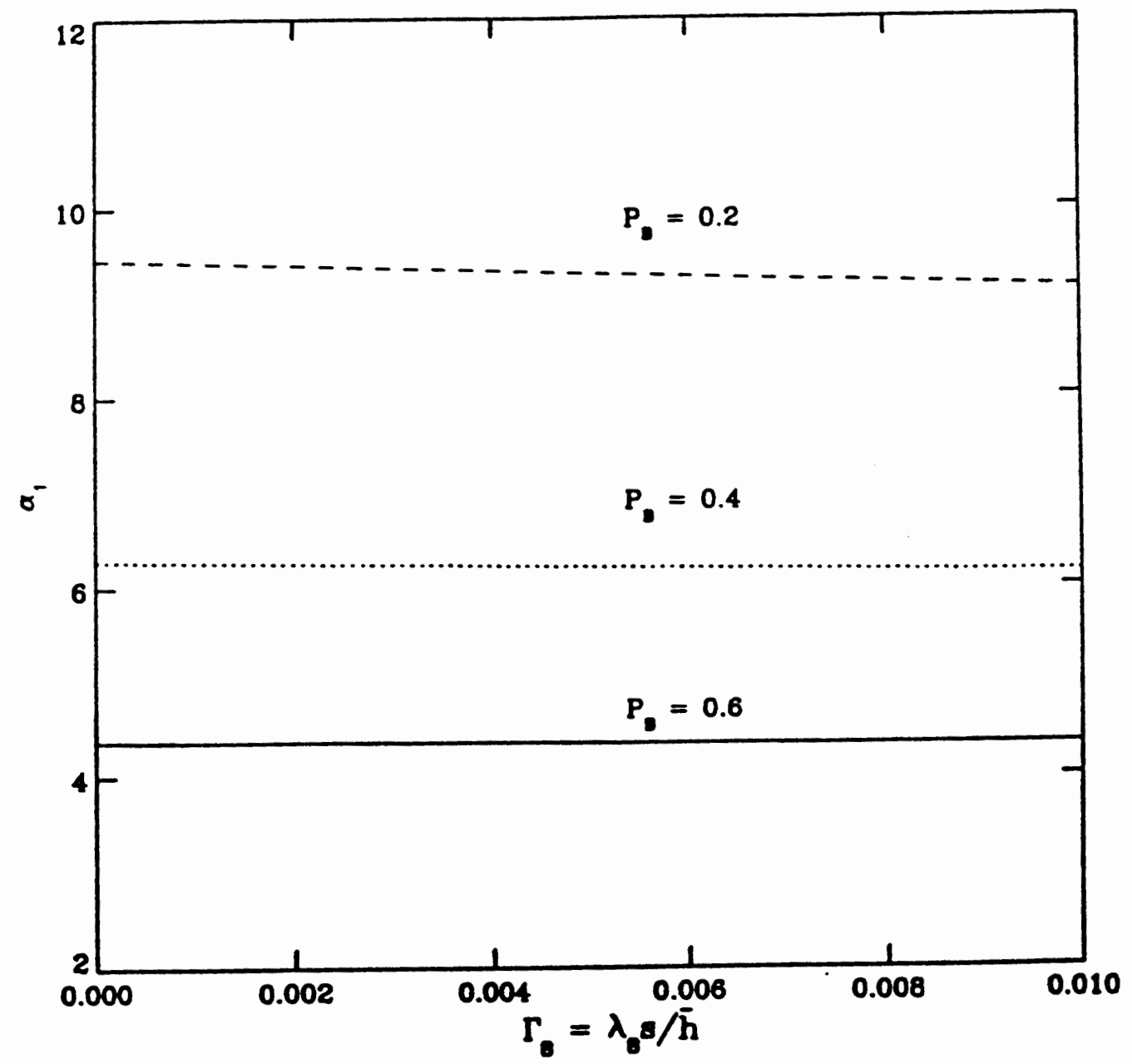

Figure 4-4: Effect of $\Gamma_{B}$ on the coefficient $\alpha_{1}$ for different $P_{B}$ 
flow conditions.

Equations (4.1) and (4.2) provide an analytical equation for predicting the effective longitudinal dispersion coefficient in a natural stream. They clearly show how stream irregularity contributes to enhanced solute dispersion and how this increase is affected by mean flow conditions. The mathematical simplicity of this expression makes it particularly attractive in practical applications.

\subsection{Effective Mean Transport Velocity}

Figures (4-5) to (4-7) shows contours of normalized effective solute transport velocity, $V_{e} / \bar{U}$, plotted against the coefficient of variation of river width, $\sigma_{B} / \bar{B}$, and bed elevation, $\sigma_{z} / \bar{h}$, for different flow Froude number. It is interesting to note that the effective solute transport velocity is always smaller than the mean flow velocity prediction based on uniformity geometry. In other words, stream nonuniformity has the effect of retarding longitudinal solute transport while enhancing solute dispersion. The retardation effect slightly increases as stream irregularity increases. In addition, unlike solute dispersion, the effective velocity seems to be more sensitive to bed elevation variation.

The geometric variability induced retardation effect is influenced by mean flow conditions, characterized the two coefficients $\beta_{1}$ and $\beta_{2}$ in Eqn. (3.71). These two coefficients can be interpreted as the relative discrepancy between effective solute transport velocity and the mean flow velocity for a unit variance in, respectively, 


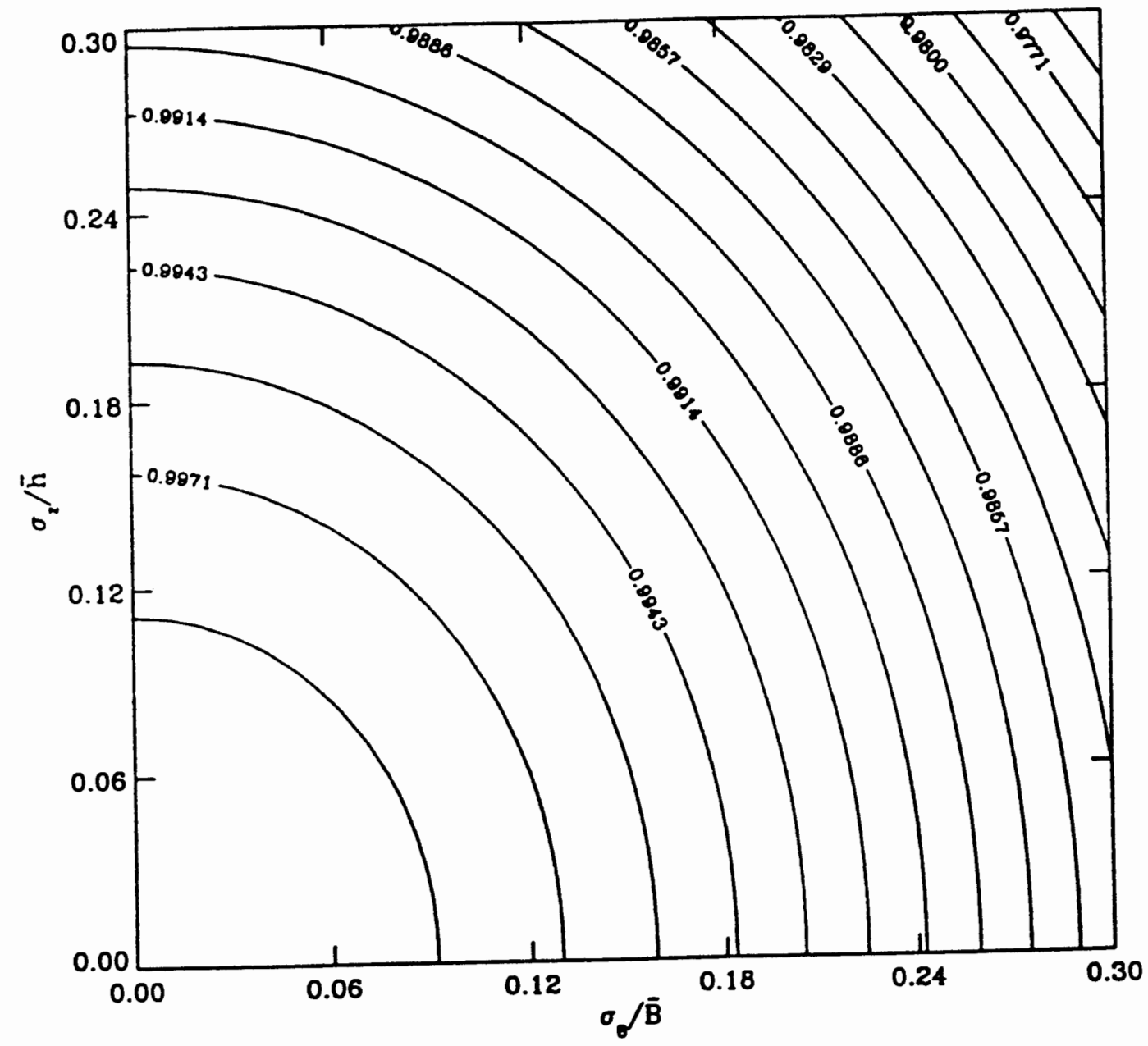

Figure 4-5: Contours of normalized effective transport velocity versus the coeffcient of variation of river width and bed elevation for $F_{r}=0.2$ 


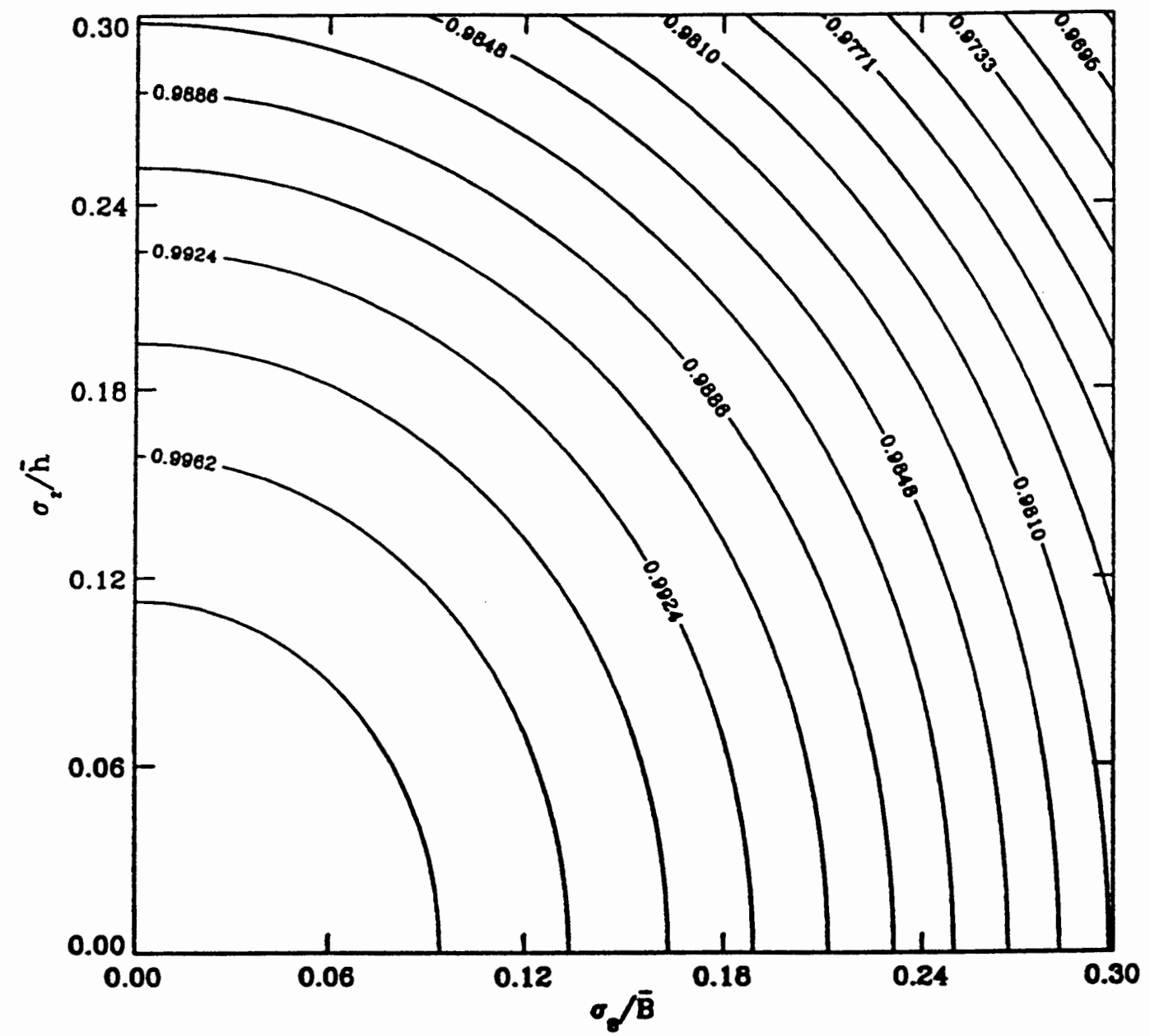

Figure 4-6: Contours of normalized effective transport velocity versus the coefficient of variation of river width and bed elevation for $F_{r}=0.4$ 


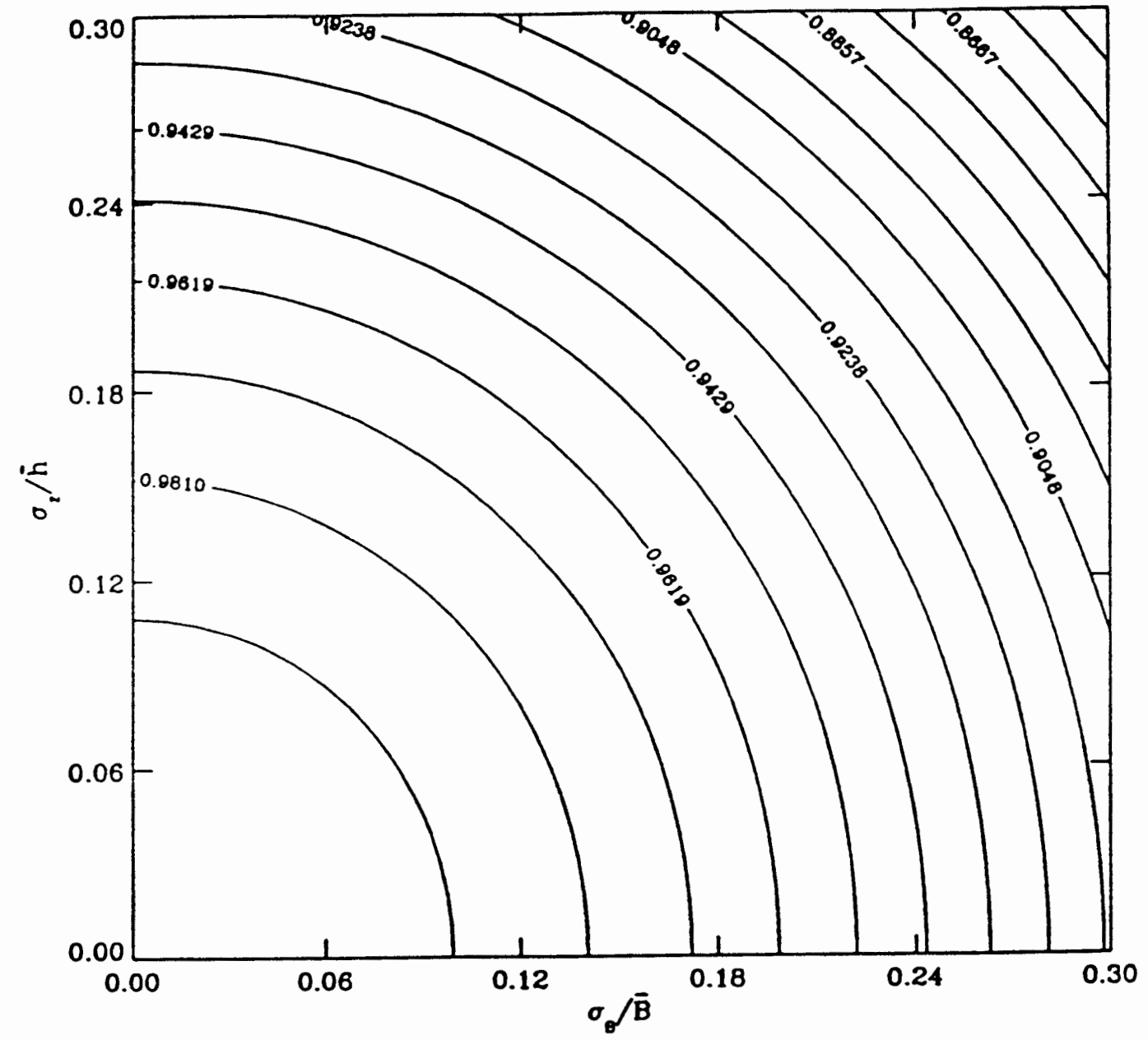

Figure 47: Contours of normalized effective transport velocity versus the coeffcient of variation of river width and bed elevation for $F_{r}=0.6$ 
river width and bed elevation. Figure (4-8) shows how $\beta_{1}$ and $\beta_{2}$ vary with the Froude number, $F_{r}$. As I can see, both $\beta_{1}$ and $\beta_{2}$ increase as the Froude number increases, with $\beta_{1}$ being slightly greater than $\beta_{2}$. This shows that the retardation effect becomes more dominant as the Froude number increases.

Figure (4-9) is the plot of $\beta_{1}$ versus $P_{B}$ and $\beta_{2}$ versus $P_{z}$, Figure (4-10) is the plot of $\beta_{1}$ versus $\Gamma_{B}$ and $\beta_{2}$ versus $\Gamma_{z}$. As It can be seen, the values of $P_{B}$ and $\Gamma_{B}$ have little effect on the value of $\beta_{1}$, and $\beta_{2}$ is also a very weak function of $\Gamma_{z}$ and $P_{z}$ for the nominal Froude number used. Sensitivity analysis for different parameter combination shows the same trend. Therefore, the expressions of $\beta_{1}$ and $\beta_{2}$ can be greatly simplified as

$$
\begin{aligned}
& \beta_{1}=\frac{\left(F_{r}^{2}-2\right)^{2}-1}{6 \pi\left(1-F_{r}^{2}\right)^{3}} \\
& \beta_{2}=\frac{1}{3 \pi\left(1-F_{r}^{2}\right)^{2}}
\end{aligned}
$$

Equations (3.75),

$$
V_{e}=\left(1-\beta_{1} \frac{\sigma_{B}^{2}}{\bar{B}^{2}}-\beta_{2} \frac{\sigma_{z}^{2}}{\bar{h}^{2}}\right) \bar{U}
$$

Eqn. (4.3) and (4.4) present a simple relationship between the effective mean solute transport velocity $V_{e}$ and the mean flow velocity. These equations clearly show how stream variability and mean flow conditions interact and dictate solute mean advection. 


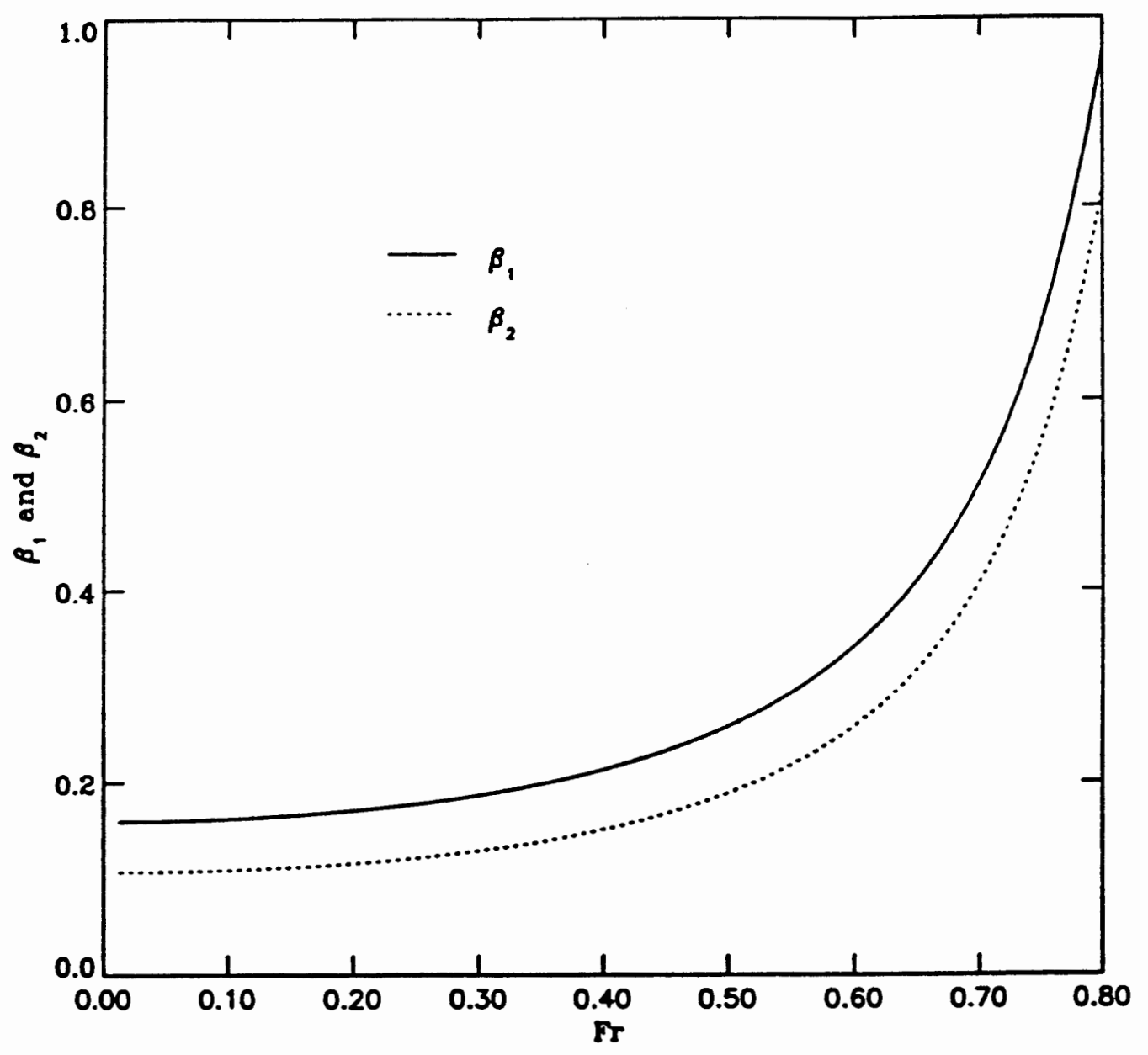

Figure 4-8: Effect of Froude number on the coefficients $\beta_{1}$ and $\beta_{2}$ 


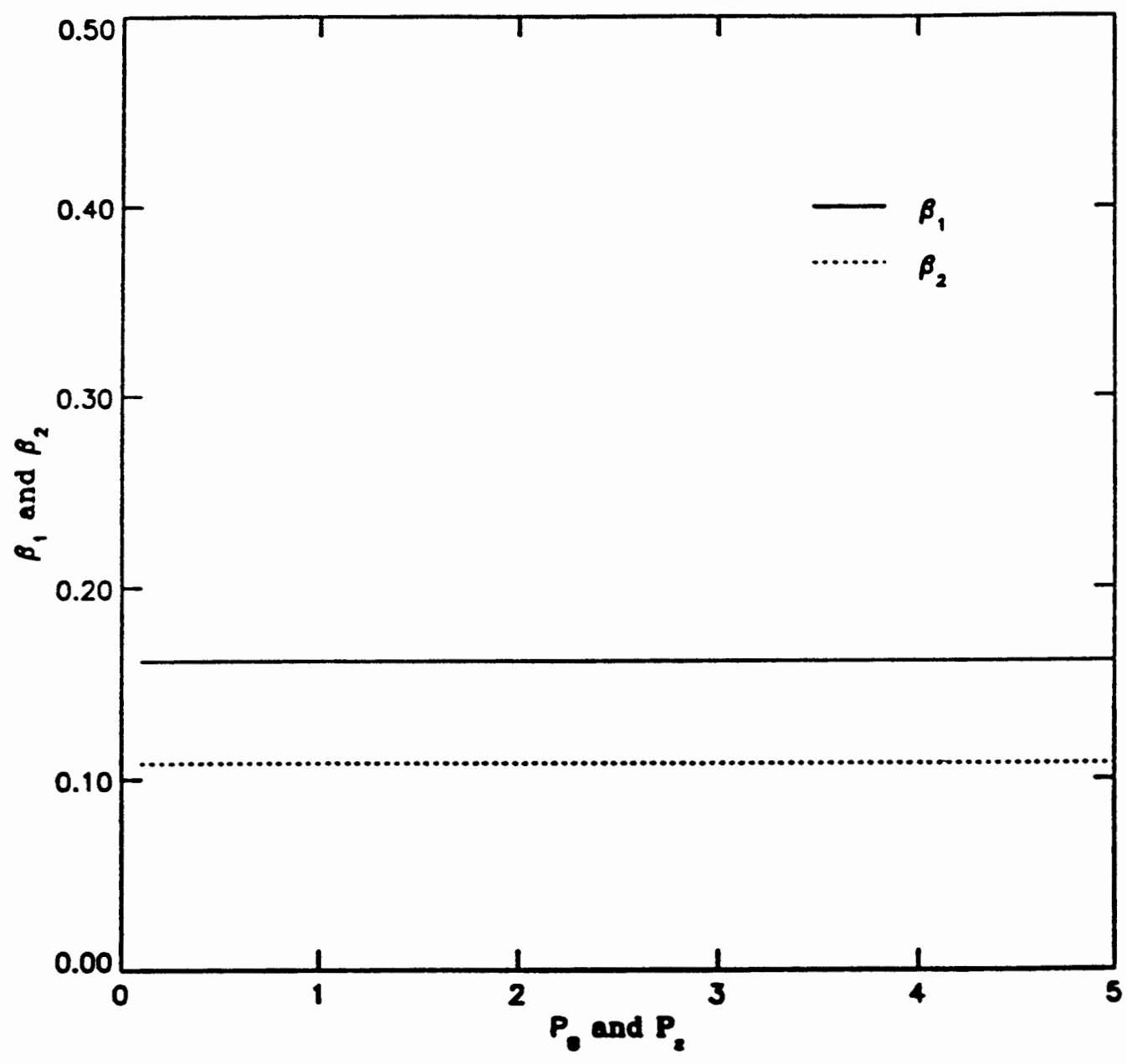

Figure 4-9: Effect of $\bar{U} \lambda_{B} / K_{0}$ (denoted as $P_{B}$ ) on the coefficient $\beta_{1}$ (solid line); Effect of $U \lambda_{3} / K_{0}$ (denoted as $P_{z}$ ) on the coefficient $\beta_{2}$ (dotted line) 


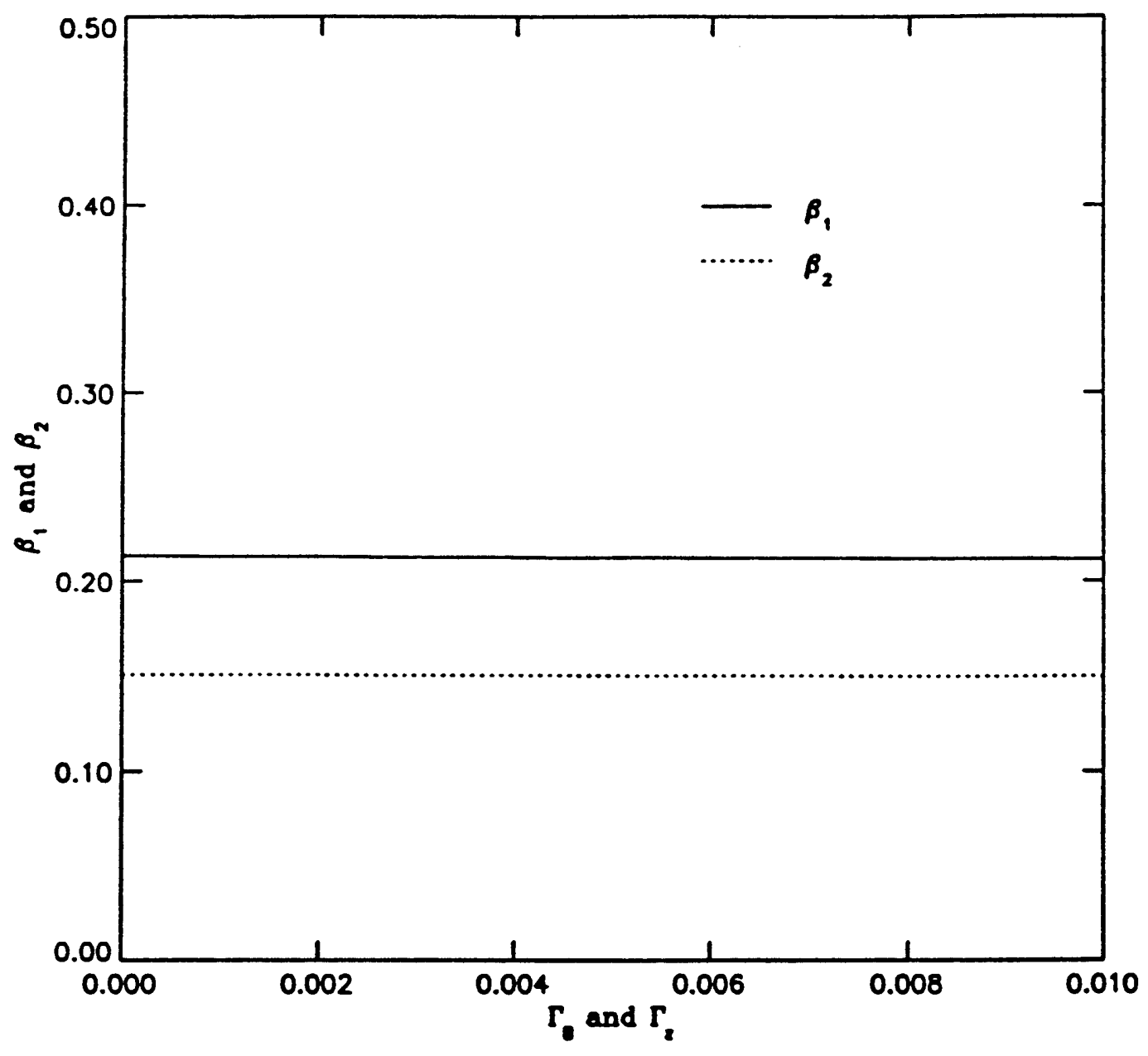

Figure 4-10: Effect of $\bar{U} \lambda_{B} / K_{0}$ (denoted as $\Gamma_{B}$ ) on the coefficient $\beta_{1}$ (solid line); Effect of $U \lambda_{z} / K_{0}$ (denoted as $\Gamma_{z}$ ) on the coefficient $\beta_{2}$ (dotted line) 


\subsection{Parameter Estimation}

Practical site-specific applications of the stochastic theory to predict effective mean transport velocity and effective longitudinal dispersion coefficient require information on the statistical parameters of longitudinal variation of river geometry, such as the standard deviations of river width, $\sigma_{B}$, and bed elevation, $\sigma_{z}$, and the correlation scales, $\lambda_{B}$ and $\lambda_{z}$. These are all physically observable characteristics, in contrast with the non-physical and empirically calibrated constants used in a conventional regression analysis. These parameters can be obtained from a statistical analysis of observed stream widths and bed elevations.

There are many ways to obtain a space series for stream width $B(x)$ and bed elevation $z(x)$ (averaged over the cross-section). One way is a direct measurement. The required data-sampling interval depends on the degree of stream irregularity. A measurement should be taken wherever there is a significant change in stream cross section. Another way to obtain stream topographical data is through remote sensing. There are various remote sensors and air photography available for stream surveys. $B(x)$ can be determined by these remote sensors and air photos. $z(x)$ can be estimated by studying the property of penetration of blue and green wavelengths through clear and turbid waters. The variances of river width and bed elevation can then be estimated directly from those detailed spatial measurements. 


\section{Chapter 5}

\section{Conclusions}

In this chapter, the major findings of this research are summarized as follows:

In this thesis a stochastic theory for analyzing longitudinal solute transport in natural streams is developed. The research represents the first attempt in predicting solute transport in real natural streams based on a rigorous analytical solution of the governing equation of solute transport in a natural stream. The theory shows that large-scale mean solute transport in an irregular, but statistically uniform, stream can be represented as a gradient diffusion process described by the classical convection dispersion equation, but using an effective solute transport velocity and an effective longitudinal dispersion coefficient.

The stochastic theory shows stream irregularity leads to "random" flow acceleration and deceleration and thus provides an additional important mixing mechanism. The effective longitudinal dispersion coefficient can be significantly greater 
than that of the corresponding uniform channel with mean stream geometry depending on the degree of river variability as well as the mean flow conditions. If the boundary variation is small and statistically uniform, the following simple and closed form expression is obtained for predicting the longitudinal dispersion coefficient:

$$
K_{e}=\left\{1+\left[\frac{8+\left(1-F_{r}^{2}\right) P_{B}^{2}}{2 \pi\left(1-F_{r}^{2}\right)^{3}}\right] \frac{\sigma_{B}^{2}}{\bar{B}^{2}}\right\} \overline{K_{0}}
$$

The stochastic theory also shows that stream irregularities tend to slow down mean solute transport. The theory predicts that the effective solute transport velocity is always smaller than the mean flow velocity. The difference increases as stream variability increases, especially when the Froude number is high. In the case that a stream is statistically uniform and under the small perturbation assumption, the following simple expression can be used to predict the effective mean solute transport velocity:

$$
V_{e}=\left\{1-\left[\frac{\left(F_{r}^{2}-2\right)^{2}-1}{6 \pi\left(1-F_{r}^{2}\right)^{3}}\right] \frac{\sigma_{B}^{2}}{\bar{B}^{2}}-\left[\frac{1}{3 \pi\left(1-F_{r}^{2}\right)^{2}}\right] \frac{\sigma_{z}^{2}}{\bar{h}^{2}}\right\} \bar{U}
$$

The results can be shown graphically by Figure (5-1). The initial plums have the same size in both natural rivers and uniform channels at time $t_{0}$. After some time the plum in a natural river is more dispersed than that in a uniform channel. However, the plum travel distance in the natural river is slightly shorter than that 

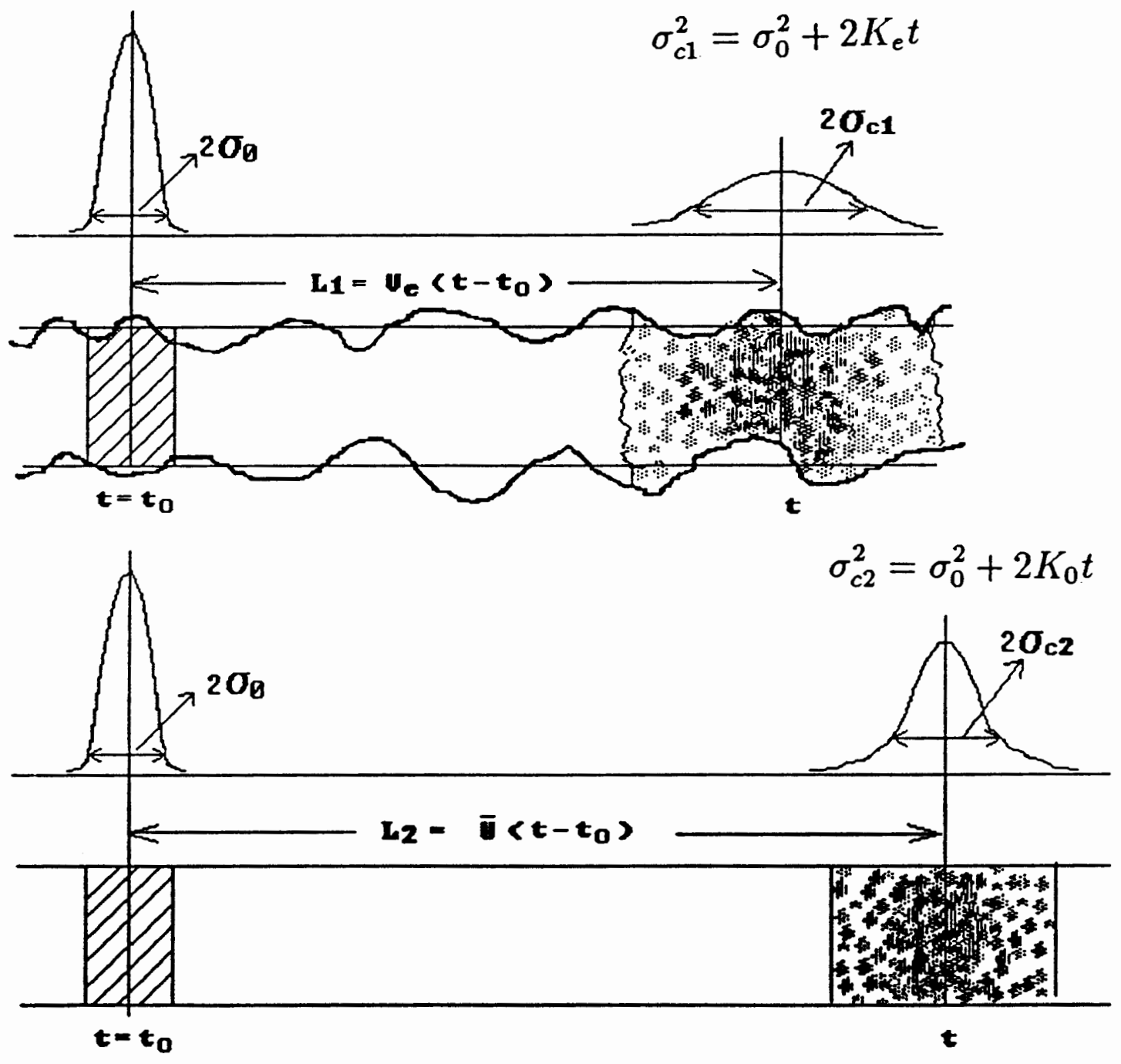

Figure 5-1: Summary of the stochastic results of solute transport in natural streams 
in the uniform channel. This shows that the river geometrical variation has the effect of enhancing longitudinal solute dispersion while slightly retarding solute transport.

It is important, however, to recognize several assumptions or limitations of this theoretical analysis when considering the field applicability of the results. In deriving the longitudinal dispersion coefficient I assumed that the mean concentration gradients in Eqns. (3.45) and (3.46) change slowly. Thus, as with all gradient-type transport relationships, Eqn. (3.61) will be strictly valid only at a large time scales when the mean concentration field is smooth.

This theoretical development also assumes statistical homogeneity of the input process $B^{\prime}(x)$ and $h^{\prime}(x)$, and constant mean width and flow depth. The assumption of stationary $B^{\prime}(x)$ and $h^{\prime}(x)$ seems to be reasonable for most naturally-formed large rivers. Many large natural streams have consistent slowly varying geometries. However, the results are obviously not applicable in the presence of isolated local irregularities caused by "dead zones", bends, man-made hydraulic structures, river islands, etc. These variations of the geometry may contain water that is significantly isolated from the main stream and serve as a storage area for waste material. Field observations or deterministic numerical simulations in these cases are then necessary. Also, for statistical homogeneity to be a meaningful concept, the overall scale of the realization must be large compared to the variability scale of the process. Thus the implication is that the theory should be applicable for transport 
over distances exceeding several variability scales. This is also required by the ergodic hypothesis invoked in the stochastic analysis.

Another important assumption is the small perturbation on $B^{\prime}(x)$ and $h^{\prime}(x)$, which is needed to obtain a closed form solution. In fact, the natural geometric variabilities of most large rivers do satisfy this requirement. In the case of large amplitude fluctuations of $B^{\prime}(x)$ and $h^{\prime}(x)$, Eqns. (5.1) and (5.2) are no longer quantitatively correct, but they can still be expected to give qualitatively correct longitudinal dispersion predictions.

This thesis is theoretical field, therefore systematic Monte Carlo simulations and field validation are needed before I can have a definitive evaluation of the applicability of this first order analysis to highly nonuniform rivers. Because of stationary geometrical variation assumption, future research needs to take the nonstationary variation of stream geometry into account. 


\section{Appendix A}

\section{Notation}

The following symbols are used in this thesis. Overbars are used for ensemble means, and primes for fluctuations about mean values.

$$
\begin{aligned}
& A=\text { cross-sectional flow area } \\
& \bar{A}=\text { ensemble mean cross-sectional flow area } \\
& A^{\prime}=\text { fluctuation of cross-sectional area } \\
& B=\text { river width } \\
& c=\text { solute concentration } \\
& d=\text { flow depth at any point in the river }
\end{aligned}
$$


$d Z_{f}=$ complex random wave amplitude for stationary random process $f(x)$

$E_{z}=$ transverse dispersion coefficient

$f \quad=$ a generic random function or random process

$F_{r} \quad=$ Froude number

$G_{1}^{\prime} \quad=$ fluctuation of concentration gradient

$G_{2}^{\prime}=$ fluctuation of second derivative of concentration

$h \quad=$ laterally averaged flow depth

$i=$ pure imaginary number, $\sqrt{-1}$

$k=$ dispersion coefficient accounting for velocity variation only within the flow cross-sectional area

$k_{0}=$ dispersion coefficient of uniform channel with mean cross-sectional $\bar{A}$ and mean flow depth $\bar{h}$

$K_{e}=$ effective longitudinal dispersion coefficient

$L=$ length of the longitudinal transport zone

$P_{B}=$ dimensionless parameter $=\lambda_{B} \bar{U} / K_{0}$

$P_{z}=$ dimensionless parameter $=\lambda_{z} \bar{U} / K_{0}$

$Q \quad=$ volumetric flow discharge

$R \quad=$ hydraulic radious

$s \quad=$ slope of river bed

$S \quad=$ power spectrum or cross spectrum 


$$
\begin{aligned}
& u^{*}=\text { shear velocity } \\
& U=\text { flow velocity at any point in the river } \\
& U^{\prime \prime}=\text { deviation of flow velocity from its cross-sectional mean } \\
& V \quad=\text { modified velocity } \\
& V_{e}=\text { effective mean velocity of solute transport } \\
& x=\text { spatial coordinate in the streamwise direction } \\
& y=\text { spatial coordinate in the transverse direction } \\
& y_{l}=\mathrm{y} \text { coordinate for the left side of river bank } \\
& y_{r}=\mathrm{y} \text { coordinate for the right side of river bank } \\
& z \quad=\text { river bed elevation } \\
& \alpha_{1}=\text { coefficient of normalized variance of river width for } K_{e} \\
& \alpha_{2}=\text { coefficient of normalized variance of river bed elevation for } K_{e} \\
& \beta_{1}=\text { coefficient of normalized variance of river width for } V_{e} \\
& \beta_{2}=\text { coefficient of normalized variance of river bed elevation for } V_{e} \\
& \zeta=\text { separation distance } \\
& \xi \quad=\text { moving coordinate system in longitudinal direction } \\
& \pi \quad=3.1415927 \ldots \\
& \lambda=\text { longitudinal variability scale of river geometric parameter }
\end{aligned}
$$


$\sigma=$ standard deviation of a stationary random process

$\tau=$ dummy variable of time integration

$\omega=$ wave number

$\Gamma_{B}=$ dimensionless parameter $=\lambda_{B} s / \bar{h}$

$\Gamma_{z}=$ dimensionless parameter $=\lambda_{z} s / \bar{h}$

$\theta_{i}=$ demensionless term defined in Eqn. (3.69)

$\psi_{i}=$ demensionless term defined in Eqn. (3.70) 


\section{Bibliography}

[1] R. Aris. On the dispersion of a solute in a fluid flowing through a tube. Proc. R. Soc. Landon Ser., A 235:67-77, 1956.

[2] K. M. Atesman. The dispersion of matter in turbulent shear flows. $\mathrm{PhD}$ thesis, Colorado state University, at Fort Collins, Colorado, 1970.

[3] M. K. Bansal. Dispersion and rearation in natural streams. $\mathrm{PhD}$ thesis, Kansas university, Lawrence, Kansas, 1970.

[4] P. C. Chatwin. On the interpretation of some longitudinal dispersion experiments. J. Fluid Mech., 48(part 4):689-702, 1971.

[5] S. C. Crow and F. H. Champagne. Orderly structure in jet turbulence. J. of Fluid Mech., 48:547-596, 1971.

[6] J. A. Cunge, F. M. Holly, and Verwey. In Practical aspects of computational river hydraulics, page 318. Pitman Publishing Limited, London, 1986. 
[7] T. J. Day. Longitudinal dispersion in natural channels. Water Resour. Res., 11(6):909-918, December 1975.

[8] J. W. Elder. The dispersion of marked fluid in turbulent shear flow. J. of Fluid Mech., Part 4:544-560, 1959.

[9] H. B. Fischer. The mechanics of dispersion in natural streams. J. of Hydr. Div., ASCE, 93(HY6):5592,187-216, November 1967.

[10] H. B. Fischer. Dispersion predictions in natural streams. J. Sanit. Div., ASCE, 94(SA5):6169, 927-943, October 1968.

[11] H. B. Fischer et al. In Mixing in inland and coastal waters. Academic press, New York, 1979.

[12] H. B. Fisher. Longitudinal dispersion and turbulent mixing in open channel flow. Rev. Fluid Mech., pages 59-77, 1973.

[13] L. W. Gelhar. In A stochastic subsurface hydrology. Prentice-Hall, Inc., New Jersey, 1993.

[14] R. G. Godfrey and B. J. Frederick. Dispersion in natural streams. USGS Prof. Paper, 433-K:1-38, 1970.

[15] A.J. Grant. A numerical model of instability in axisymmetric jets. J. of Fluid Mech., 66:707-724, 1974. 
[16] E. Hirst. Zone of flow establishment for round buoyant jets. Water Resour. Res., 8:1234-1246, 1972.

[17] K. M. Kizer. Material and momentum transport in axisymmetric turbulent jets of water. Am. Inst. Chem. Eng. J., 9:386-390, 1963.

[18] S. G. Li, L. Venkataraman and D. McLaughlin. Stochastic theory for irregular stream modeling. part i: Flow resistance. J. of Hydr. Eng., 118(8):1079-1090, August 1992.

[19] H. Liu. Predicting dispersion coefficient of streams. J. of Env. Eng. Div., Proc. ASCE, 103(EE1):59-69, February 1977.

[20] J. L. Lumley and H. A. Panofsky. In The structure of atmospheric turbulence. John Wiley, New York, 1964.

[21] R. S. McQuivey and T. Keefer. Simple method for predicting dispersion in streams. J. Environ. Eng. Div., Proc. Am. Soc. Civ. Eng., 100:997-1011, 1974.

[22] A. C. Miller. Diffusion and dispersion in open channel flow. J. Hydraul. Div., Proc. Am. Soc. Civ. Eng., 100:159-171, 1974.

[23] C. F. Nordin and B. V. Sabol. Empirical data on longitudinal dispersion in rivers. USGS Water Resource investigation, pages 20-24, 1974. 
[24] M. B. Priestley. In Spectral analysis and time series, volume 2: Multivariate series, prediction and control, page part B. Academic press, New York, 1981.

[25] W. W. Sayre and F. M. Chang. A laboratory investigation of the open channel dispersion process for dissolved, suspended, and floating dispersants. USGS Prof. Paper, (433-E), 1968.

[26] A. A. Sooky. Longitudinal dispersion in open channels. J. Hydr. Div. ASCE, 95(HY4):1327-1346, July 1969.

[27] G. I. Taylor. Diffusion by continuous movements. Proc. London Math. Soc. Ser. A, 20:196-211, 1921.

[28] G. I. Taylor. Dispersion of solute matter in solvent flowing through a tube. Proc. Roy. Soc. Ser. A, 219:186-203, 1953.

[29] G. I. Taylor. The dispersion of matter in turbulent flow through a pipe. Proc. R. Soc. London Ser. A, 223:446-468, 1954.

[30] E. L. Thackston and P. A. Krenkel. Longitudinal mixing in natural streams. J. Sanit Eng. Div., ASCE, 93(SA5):67-90, October 1967. 